\title{
Statistical characteristics of large scale structure ${ }^{\star}$
}

\author{
M. Demiański ${ }^{1,2}$ and A. G. Doroshkevich ${ }^{3,4}$ \\ 1 Institute of Theoretical Physics, University of Warsaw, 00-681 Warsaw, Poland \\ 2 Department of Astronomy, Williams College, Williamstown, MA 01267, USA \\ 3 Theoretical Astrophysics Center, Juliane Maries Vej 30, 2100 Copenhagen Ø, Denmark \\ e-mail: dorr@tac.dk \\ 4 Keldysh Institute of Applied Mathematics, Russian Academy of Sciences, 125047 Moscow, Russia
}

Received 2 May 2003 / Accepted 19 April 2004

\begin{abstract}
We generalize and extend the statistical description of formation and evolution of Large Scale matter distribution in the Universe proposed in our previous papers. We investigate the impact of transverse motions - expansion and/or compression - on properties of the Large Scale Structure elements - walls, pancakes, filaments and clouds - and, generalizing the Press-Schechter formalism, derive their mass functions. Using the Zel'dovich theory of gravitational instability we show that these mass functions are approximately the same and the mass of each type of elements is found to be concentrated near the corresponding mean mass. At high redshifts, both the mass function and the mean mass of formed elements depend upon the small scale part of the initial power spectrum and, in particular, upon the mass of dominant fraction of dark matter (DM) particles. Using these results we obtain independent estimates of probable redshifts of the reionization and reheating periods of the Universe. We show that the transverse motions do not significantly change the redshift evolution of the observed mass function and the mean linear number density of low mass pancakes related to absorption lines in the spectra of the farthest quasars. Application of this approach to the observed Lyman $-\alpha$ forest allows one to directly estimate the shape of initial power spectrum on small scales.
\end{abstract}

Key words. cosmology: large-scale structure of Universe - cosmology: dark matter - galaxies: formation

\section{Introduction}

In recent years numerical simulations are being more often used to investigate the process of formation and evolution of the Large Scale Structure (LSS) of the Universe. This trend is strongly stimulated by the rapid growth of computer facilities and it provides better and better high quality simulations (see e.g., Benson et al. 2001; Smith et al. 2003; Frenk 2002). Modern simulations performed in large boxes and with high resolution can take into account simultaneous action of many important physical factors and they substantially increase available information about the process of LSS formation and evolution.

At small redshifts the LSS is observed in the large galaxy surveys as a system of filaments and walls (see, e.g., Zel'dovich et al. 1982; Einasto et al. 1984; de Lapparent et al. 1986). At high redshifts the LSS is observed mainly through the distribution of the Lyman- $\alpha$ clouds identified through absorption lines in spectra of the farthest quasars. All these elements of the LSS are formed by mildly nonlinear processes and, in contrast with galaxies and clusters of galaxies, they are relaxed along the shorter axes only. They are quite well reproduced in numerical

^ Appendices are only available in electronic form at http://www. edpsciences.org simulations, what indicates their close relation with the initial power spectrum. However, this relation is not yet well understood and a quantitative description of the LSS elements only recently got under way. Now the analysis of both simulated and observed catalogs of galaxies is mainly focused on the discussion of the correlation function, $\xi(r)$, and the power spectrum, $p(k)$, while other characteristics of the LSS are not determined. This limited description demonstrates that numerical simulations cannot substitute theoretical models of structure formation that provide the basis for much more detailed quantitative description of both the observed and simulated large scale matter distribution.

Theoretical models reveal the main factors that influence the process of structure formation and evolution and clarify links between them and the measured characteristics of the LSS. This opened up a possibility of formulating new and promising approaches of statistical description of both simulated and observed characteristics of the LSS such as, for example, a set of mass functions of structure elements, their separations and so on. These characteristics can be measured with such powerful methods as the Minimal Spanning Tree (MST) technique (Barrow et al. 1985; van de Weygaert 1991; Demiański et al. 2000, hereafter DDMT; Doroshkevich et al. 2001, 2004a,b for the SDSS and 2dF surveys) and 
the Minkowski Functional method (see, e.g., Mecke et al. 1994; Kerscher 2000; Shandarin et al. 2003). Some others methods had been discussed in Shandarin (1983), Babul \& Starkman (1992), Luo et al. (1996), Martinez \& Saar (2002). The high efficiency of such approaches is illustrated by the Press-Schechter relation (Press \& Schechter 1974) for the mass function of high density clouds which has been widely used and discussed during the last thirty years (see, e.g., Mo \& White 1996; Loeb \& Barkana 2001; Sheth \& Tormen 2002; Scannapieco \& Barkana 2002).

Some statistical characteristics of the LSS have been derived in our previous papers (Demiański \& Doroshkevich 1999, hereafter DD99; DDMT). They are based on the nonlinear theory of gravitational instability (Zel'dovich 1970; Shandarin \& Zel'dovich 1989; for review, Sahni \& Coles 1995) applied to a CDM-like broad band initial power spectrum of perturbations. This approach allows to outline the general tendencies of the LSS evolution and demonstrates the leading role of the initial velocity field and of the successive merging of structure elements in the process of formation of large scale matter distribution. Its efficiency and limitations were tested on simulated DM distributions and mock catalogs (see, e.g., DDMT, Doroshkevich et al. 2004a,b).

Indeed, the well known large difference between the coherent lengths of initial density and velocity fields - at least two orders of magnitude (Sect. 2.1) - indicates that the formation of the LSS elements is driven mainly by the velocity field. Using the velocity perturbations the Zel'dovich theory quite successfully describes the process of structure formation in the DM component up to the moment of caustic creation. Both the Zel'dovich theory and our approach do not describe the evolution of compressed matter such as the processes of violent relaxation, the disruption of the LSS elements to the system of high density clouds and so on. However, these processes do not distort the integral characteristics of the LSS such as the fraction of matter accumulated within filaments and pancakes or walls, their number density, the surface density of walls and linear density of filaments, their mass functions, velocities of each element as a whole and velocity dispersion within relaxed elements and some others. These stable statistical characteristics of the LSS are mainly defined by the process of formation of LSS elements and can be derived from the initial power spectrum and cosmological model at all evolutionary stages. Here we consider only such characteristics.

The Zel'dovich theory and our approach do not consider the evolution of baryonic component and accompanied thermal and gasdynamic processes important for the galaxy formation (see, e.g., Rees 1985; Rees \& Dekel 1986; Dekel \& Silk 1987) and for the appearance of LSS at high redshifts. In all these cases, the observed characteristics of baryonic and DM components are different and to link them special analysis is called for. We do not discuss these problems here. Even so, the application of our statistical approach to the observed galaxy surveys (Doroshkevich et al. 2004a,b) and Ly- $\alpha$ forest (Demiański et al. 2003a,b) demonstrate high efficiency of the proposed method for interpretation of the observed characteristics of the LSS.

In contrast with many previously discussed models of the LSS formation such as, for example, the peak - patch picture of Bond \& Myers (1996) and the model of Bond et al. (1996), our approach allows one to find the statistical characteristics of the LSS elements without any artificial smoothing of the initial fields. It demonstrates that the possible feedback of formation of galaxies and even cluster of galaxies on the evolution of the LSS is quite moderate because of the small fraction of matter accumulated within such objects.

The Zel'dovich theory describes the process of LSS formation as a successive creation of pancakes, their further compression into filaments and filaments into clouds with a progressive growth of the masses and sizes of structure elements due to their merging. It correctly describes properties of pancakes and walls formed owing to the 1D collapse, but underestimates the rate of collapse and overestimates the expansion rate after caustic formation. This means that our approach decelerates the process of formation of filaments and clouds, and our estimates of their masses and the matter fractions assigned to them correspond to earlier stages of their evolution as compared with observations and simulations. The properties of clouds are also changing in the course of their evolution within the filaments and walls. The same problems appear in the Press-Schechter formalism and its extensions. These relations can be improved after detailed comparison with numerical simulations.

Bearing in mind these comments, we focus our attention on the discussion of pancakes and walls while only some basic characteristics of filaments and clouds are considered. Physical aspects of our approach and main results are also discussed in Sect. 8.

Comparison of the theoretical expectations with measured statistical parameters of the most conspicuous walllike component of structure was performed in DDMT and in Doroshkevich et al. (2004a,b) for the mock 2dF survey (Cole et al. 1998) and the SDSS Data Release 1 (Stoughton et al. 2002; Abazajian et al. 2003) and 2dF (Colless et al. 2003). The Las Campanas Redshift Survey (Shectman et al. 1996) was considered in Doroshkevich et al. (2001). These investigations confirm that the walls are gravitationally confined and partly relaxed Zel'dovich pancakes formed presumably due to the 1D collapse of matter. Such interpretation was already proposed in Thompson \& Gregory (1978) and Oort (1983) just after the first walls have been observed.

From this comparison it follows that the main measured characteristics of both simulated and observed walls are consistent with the theoretical expectations. They are quite well expressed in terms of the time scale, the coherent length and correlation functions of the initial velocity field set by the power spectrum of initial perturbations. The time scale and the coherent length are expressed through the spectral moments and amplitude of the initial perturbations, and the basic parameters of the cosmological model. This analysis provides independent estimates of the amplitude of initial perturbations with a scatter $\leq 20 \%$ and verifies their Gaussianity.

Results obtained in DD99 and DDMT provide a reliable base for further investigations but impact of some important factors was not considered in these papers. Here we extend these models by taking into account the action of some factors responsible for the evolution of structure elements after their formation. Among the most important are: the expansion and 
compression of LSS elements in the transverse directions, the small scale damping of initial perturbations caused by the random motions of DM particles and Jeans damping, and the acceleration of cloud formation within larger structure elements, what creates a large scale bias between the spatial distribution of DM and luminous matter.

First of all, considering the expansion and compression of LSS elements in the transverse directions, we approximate the rates of formation of LSS elements and their mass functions in a wide range of redshifts. For all LSS elements - pancakes, filaments and high density clouds - the mass functions are found to be quite similar to each other. They are also similar to those predicted by the Press-Schechter formalism for a suitable initial power spectrum (Loeb \& Barkana 2002) and its extension for elliptical clouds (Sheth \& Tormen 2002). This similarity demonstrates the generic dependence of the characteristics of the LSS on the initial power spectrum and indicates that the shape of collapsed clouds influences the rate of collapse but does not change significantly the mass functions. These results complement the Press-Schechter approach and allow one to obtain independent estimates of the mass functions for a wider class of objects.

The same factors change the basic properties of long-lived pancakes observed as the Ly- $\alpha$ forest in a wide range of redshifts (see, e.g., Cen et al. 1994; Dave et al. 1999). In this paper we discuss the evolution of two most important characteristics of such pancakes: their surface density and their mean number density along the line of sight. Both characteristics are evidently changing with redshift due to the formation of new and merging of old pancakes, and their transverse compression and expansion. Both characteristics play a key role in the discussion of observed evolution of the Ly- $\alpha$ forest and we used them (Demiański et al. 2003) to show that the observed properties of absorbers are consistent with theoretical expectations. This approach also allows one to measure the shape of initial power spectrum down to scales of $\sim 30 h^{-1} \mathrm{kpc}$ (Demiański \& Doroshkevich 2003). It is important to notice that the surface density of pancakes is sensitive to the model of absorbers used in the analysis while the mean number density along the line of sight depends only on the position of absorbers and is independent from the physical state of the intergalactic matter and the model of absorbers.

The properties of LSS are also strongly influenced by the interaction of small and large scale perturbations what accelerates or decelerates formation of less massive clouds within compressed or expanded LSS elements. In simulations this interaction manifests itself by a strong concentration of halos within filaments and walls at all redshifts (see, e.g., Mo \& White 1996; Lemson \& Kauffmann 1999; Sheth \& Tormen 1999, 2002; Gottlöeber et al. 2001, 2002, 2003; Sect. 5.7). In observations it is seen as a strong concentration of galaxies within the richer filaments and walls and as an essential difference in richness of galaxy groups situated within walls and filaments (see, e.g., Doroshkevich et al. 2004a,b). In contrast, a significant fraction of both DM and baryons remains within low mass pancakes that are more homogeneously distributed than the luminous matter and are observed as the Ly- $\alpha$ forest. We show that the formation of high density halos and galaxies is modulated by the large scale initial velocity field what explains qualitatively the large scale bias. The approach used in this paper allows one to demonstrate and roughly quantify this dependence.

However, the fragmentation of the LSS elements into the system of high density clouds and their further evolution cannot be described with our approach. The analysis of simulations (see, e.g., Gottlöber et al. 2001, 2002, 2003) indicates that the quantitative description of these processes requires special models.

This paper is organized as follows: in Sect. 2 basic relations are introduced. In Sect. 3 the redshift dependence of the expected matter fraction assigned to various types of structure elements is found. In Sect. 4 we discuss the interaction of large and small scale perturbations. In Sect. 5 the joint mass functions of DM structure elements are considered. In Sects. 6 and 7 we discuss the redshift evolution of statistical characteristics of filaments and pancakes. Short conclusions can be found in Sect. 8. Some technical details are given in Appendices A and $\mathrm{B}$.

\section{Basic statistical characteristics of the Zel'dovich theory}

In this section we present the basic statistical characteristics of Zel'dovich approximate nonlinear theory of gravitational instability used later to describe the process of structure formation and evolution. Main ideas and characteristics were already introduced in DD99 and are repeated here without discussion. Here we generalize and extend this description that allows us to extend its application. Some definitions are improved and corrected what makes the approach more transparent.

In the Zel'dovich theory (Zel'dovich 1970; Shandarin \& Zel'dovich 1989) the Eulerian, $r_{i}$, and the Lagrangian, $\tilde{q}_{i}$, coordinates of particles (fluid elements) are related by

$r_{i}=(1+z)^{-1}\left[\tilde{q}_{i}-B(z) S_{i}(\tilde{\boldsymbol{q}})\right]$,

where $z$ denotes the redshift, $B(z)$ describes growth of perturbations in the linear theory, and the gradient vector $S_{i}(\tilde{\boldsymbol{q}})=$ $\partial \phi / \partial \tilde{q}_{i}$ characterizes the spatial distribution of perturbations. The Lagrangian coordinates of a particle, $\tilde{q}_{i}$, are its unperturbed coordinates in the real space, $r_{i}(z=0)=\tilde{q}_{i}$. For the spatially flat $\Lambda \mathrm{CDM}$ cosmological model the function $B(z)$ can be approximated (DD99) as follows:

$B^{-3}(z) \approx \frac{\Omega_{\Lambda}+2.2 \Omega_{\mathrm{m}}(1+z)^{3}}{1+1.2 \Omega_{\mathrm{m}}}, \Omega_{\Lambda}+\Omega_{\mathrm{m}}=1$.

Here $\Omega_{\mathrm{m}}$ and $\Omega_{\Lambda}$ are the dimensionless matter density and cosmological constant. This fit is reasonably accurate for all $z$ and is normalized at $z=0$ by the condition $B(0)=1$.

In this paper we consider only power spectra with the Harrison-Zel'dovich asymptotic, $p(k) \propto k$, at $k \rightarrow 0$, and CDM-like or WDM-like transfer functions, $T^{2}(k)$, introduced in Bardeen et al. (1986, hereafter BBKS):

$p(k)=\frac{A^{2} k}{4 \pi k_{0}^{4}} T^{2}\left(\frac{k}{k_{0}}\right), \quad k_{0}=\Omega_{\mathrm{m}} h^{2} \mathrm{Mpc}^{-1}$, 
where $A$ and $h=H_{0} / 100 \mathrm{~km} \mathrm{~s}^{-1} \mathrm{Mpc}^{-1}$ are the dimensionless amplitude of perturbations and the Hubble constant, $k$ is the comoving wave number. The same approach can be applied for arbitrary initial power spectra.

\subsection{Coherent lengths and correlation functions of initial density and velocity fields}

For the spectra (3), the coherent lengths of initial density and velocity (or displacement) fields, $l_{\rho}$ and $l_{v}$, are expressed through the spectral moments, namely, $m_{-2}$ and $m_{0}$, (DD99):

$m_{0}=\int_{0}^{\infty} x^{3} T^{2}(x) D_{W}(x) D_{J}(x) \mathrm{d} x$,

$m_{-2}=\int_{0}^{\infty} x T^{2}(x) \mathrm{d} x \approx 0.023, \quad x=k / k_{0}$,

$l_{v}=\frac{1}{k_{0} \sqrt{m_{-2}}} \approx \frac{6.6}{\Omega_{\mathrm{m}} h^{2}} \mathrm{Mpc}, \quad l_{\rho}=q_{0} l_{v}=\frac{5}{k_{0}} \frac{\mathrm{m}_{-2}^{3 / 2}}{m_{0}}$.

Here $D_{\mathrm{W}}$ and $D_{\mathrm{J}}$ describe damping of perturbations caused by the random motions of DM particles (see BBKS) and suppression of formation of baryonic clouds due to gaseous pressure (Jeans damping, see, e.g., Matarrese \& Mohayaee 2002), respectively. Analysis of simulations and observed Ly- $\alpha$ forest (Narayanan et al. 2000; Barkana et al. 2001; Demiański et al. 2003; Demiański \& Doroshkevich 2003) allows to estimate $q_{0}$ and $M_{\mathrm{DM}}$ as follows:

$M_{\mathrm{DM}} \geq 1-5 \mathrm{keV}, \quad q_{0} \leq 10^{-2}$.

The characteristic masses of DM clouds associated with the coherent lengths $l_{v}$ and $l_{\rho}$ are

$M_{v}=\frac{4 \pi}{3}\langle\rho\rangle l_{v}^{3} \approx \frac{2 \times 10^{14}}{\left(\Omega_{\mathrm{m}} h^{2}\right)^{2}} M_{\odot}, \quad M_{\rho}=q_{0}^{3} M_{v}$.

Large difference between the coherent lengths $l_{v}$ and $l_{\rho}$ indicates that formation of galaxy filaments and walls observed at small redshifts is mainly driven by the larger coherent length, $l_{v}$. The small scale correlations of density field characterized by the coherent length $l_{\rho}$ are more important during formation, at high redshifts, of low mass objects such as the first population of galaxies and Ly- $\alpha$ absorbers.

For spectra under consideration, the normalized correlation functions of displacement and density fields,

$\xi_{v}(q)=3 \frac{\left\langle\left(\boldsymbol{q} \cdot \boldsymbol{S}\left(\tilde{\boldsymbol{q}}_{1}\right)\right)\left(\boldsymbol{q} \cdot \boldsymbol{S}\left(\tilde{\boldsymbol{q}}_{2}\right)\right)\right\rangle}{\sigma_{s}^{2} q^{2}}, \quad \sigma_{s}^{2}=\frac{A^{2} m_{-2}}{k_{0}^{2}}$,

$\xi_{\rho}(q)=\frac{\left\langle\rho\left(\tilde{\boldsymbol{q}}_{1}\right) \rho\left(\tilde{\boldsymbol{q}}_{2}\right)\right\rangle-\langle\rho\rangle^{2}}{\langle\rho\rangle^{2} \sigma_{\rho}^{2}}, \quad \sigma_{\rho}^{2}=A^{2} m_{0}$,

$\boldsymbol{q}=\left(\tilde{\boldsymbol{q}}_{1}-\tilde{\boldsymbol{q}}_{2}\right) / l_{v}, \quad q=\left|\tilde{\boldsymbol{q}}_{1}-\tilde{\boldsymbol{q}}_{2}\right| / l_{v}$,

were approximated in DD99. Here, as before, $\tilde{\boldsymbol{q}}_{1}$ and $\tilde{\boldsymbol{q}}_{2}$ are real unperturbed coordinates of two particles at $z=0, \sigma_{s}^{2}$ and $\sigma_{\rho}^{2}$ are variances of the displacement and the density fields, and $A$ is the amplitude of initial perturbations introduced in Eq. (3). Due to homogeneity and isotropy of both the background matter distribution and perturbations the correlation functions depend only on the differences of coordinates $|\boldsymbol{q}|$. For the most interesting case $q_{0} \ll 1, q \leq 1$, the correlation functions can be written as follows:

$\xi_{v} \approx 1-\frac{q^{2}}{\sqrt{q^{2}+q_{0}^{2}}} \frac{q_{0}+2 \sqrt{q^{2}+q_{0}^{2}}}{q_{0}+\sqrt{q^{2}+q_{0}^{2}}}$,

$\xi_{\rho}=-\frac{q_{0}}{15} \frac{1}{q^{4}} \frac{\mathrm{d}}{\mathrm{d} q} q^{4} \frac{\mathrm{d} \xi_{v}}{\mathrm{~d} q} \approx \frac{q_{0}}{\sqrt{q_{0}^{2}+q^{2}}}$, for $q \leq q_{0} \ll 1$,

and $\xi_{\rho} \ll 1$, for $q_{0} \leq q$. At $q \ll q_{0}$ both functions, $\xi_{v}$ and $\xi_{\rho}$, only weakly depend upon the higher spectral moments, $m_{2}, m_{4}, \ldots$. Some of the more cumbersome correlation and structure functions of perturbations are discussed in DD99 and are presented in the Appendix A.

\subsection{Basic relations}

As was shown in DD99, to describe the formation of structure elements the basic Eq. (1) has to be rewritten using the differences of particle coordinates and displacements. The separation of two particles with Lagrangian and Euler coordinates $\tilde{\boldsymbol{q}}_{1}$ and $\tilde{\boldsymbol{q}}_{2}$, and $\boldsymbol{r}_{1}$ and $\boldsymbol{r}_{2}$, respectively, is described by the equations $(i=1,2,3)$ :

$\Delta r_{i}=\left(\boldsymbol{r}_{1}-\boldsymbol{r}_{2}\right)_{i}=\frac{l_{v}}{1+z}\left[q_{i}-\frac{B \Delta S_{i}\left(q_{i}\right)}{l_{v}}\right]$,

$\Delta S_{i}=S_{i}\left(\tilde{\boldsymbol{q}}_{1} / l_{v}\right)-S_{i}\left(\tilde{\boldsymbol{q}}_{2} / l_{v}\right), \quad q_{i}=\frac{\left(\tilde{\boldsymbol{q}}_{1}-\tilde{\boldsymbol{q}}_{2}\right)_{i}}{l_{v}}$

Evidently, for Gaussian initial perturbations, the PDF of $\Delta S_{i}$ is also Gaussian with

$\left\langle\Delta S_{i}\left(q_{i}\right)\right\rangle=0, \quad \frac{3}{\sigma_{s}^{2}}\left\langle\Delta S_{i}^{2}\left(q_{i}\right)\right\rangle=\sigma_{q}^{2}=2\left[1-\xi_{v}\left(q_{i}\right)\right]$.

These relations show the symmetry between expansion and compression of matter and the progressive growth of $\left\langle\Delta S_{i}^{2}\left(q_{i}\right)\right\rangle \propto\left|q_{i}\right|$ for larger $\left|q_{i}\right|\left(1 \geq\left|q_{i}\right| \gg q_{0}\right)$. These expressions indicate also a relatively slower rate of evolution of objects with larger $\left|q_{i}\right|$, because it is defined by the ratio $\Delta S_{i} /\left|q_{i}\right| \propto \sigma_{q} / q \propto q^{-1 / 2}$.

According to the relations (10), when two particles with different Lagrangian coordinates $\tilde{\boldsymbol{q}}_{1}$ and $\tilde{\boldsymbol{q}}_{2}$ meet at the same Eulerian point $\boldsymbol{r}$ a caustic - Zel'dovich pancake - with the surface mass density $<\rho>\left|\tilde{\boldsymbol{q}}_{1}-\tilde{\boldsymbol{q}}_{2}\right|$ forms. Following DD99 we assume that all particles situated between these two boundary particles are also incorporated into the same pancake. This assumption is also used in the adhesion approach (see, e.g., Shandarin \& Zel'dovich 1989). Comparison of statistical characteristics of pancakes with simulations (DD99; DDMT) verifies this approach and shows that long lived and partly relaxed richer walls accumulate a significant fraction of compressed matter. The same condition can be used to approximate formation of filaments and clouds in the course of successive collapse along two and three axes. 
For the general description of structure formation it is convenient to combine the three Eqs. (10) into one scalar equation, namely,

$q_{i} \Delta r_{i}=\frac{l_{v} q^{2}}{1+z}[1-\tau(z) F(\boldsymbol{q})]$

$\tau(z)=\frac{\sigma_{s}}{\sqrt{3} l_{v}} B(z)=\tau_{0} B(z), \quad F(\boldsymbol{q})=\frac{\sqrt{3} \boldsymbol{q} \Delta \boldsymbol{S}(\boldsymbol{q})}{\sigma_{s} q^{2}}$.

The random function $F(\boldsymbol{q})$ with the Gaussian PDF and

$\langle F\rangle=0, \quad\left\langle F^{2}\right\rangle=\sigma_{F}^{2}(\boldsymbol{q})$,

characterizes the evolution of a region defined by the given limiting o $\boldsymbol{q}$ 's. The dispersion $\sigma_{F}(\boldsymbol{q})$ is expressed through the structure functions introduced in Appendix A and DD99. The conditions $F(\boldsymbol{q}) \geq 1$ and $F(\boldsymbol{q}) \leq 0$ separate the collapsed and expanded parts of the volume, the condition $0 \leq F(\boldsymbol{q}) \leq 1$ separates part of the volume that will collapse later. This approach allows one to obtain the main characteristics of structure. However, its practical applications are not simple owing to the complicated form of the function $\sigma_{F}(\boldsymbol{q})$.

\subsection{Characteristics of the deformation field}

In DD99 we assumed that the deformation field is dominated by two lowest harmonics what allows one to characterize the formation of structure by three weakly correlated components of the displacements. Analysis performed in Appendix B shows that, for the spectra (3), this assumption is valid with a precision better than $10 \%$. For the most interesting cases $q_{0} \ll q \ll 1$ and $q \ll q_{0} \ll 1$, the normalized amplitudes of several lowest spherical harmonics of the deformation field, $b_{l}^{2}$, are given by:

$b_{0}^{2} \approx 0.533, b_{2}^{2} \approx 0.381, \quad b_{4}^{2} \approx 0.037, b_{6}^{2} \approx 0.014, \ldots$

$b_{0}^{2} \approx 0.55, \quad b_{2}^{2} \approx 0.44 \gg b_{4}^{2}, \quad b_{6}^{2} \cdots$

and the contribution of higher order harmonics with $l \geq 4$ is only $\sim 1 \%$ for low mass clouds with $q \leq q_{0} \ll 1$, and it reaches $\sim 8 \%$ for more massive clouds with $q_{0} \ll q \leq 1$.

These results justify the assumption made in DD99 to neglect higher order harmonics of perturbations with $l \geq 4$ and they confirm that the formation of structure can be approximately described by the spherical and quadrupole components of the deformation field. The influence of higher harmonics, even with small amplitude, leads to a small scale disruption of the compressed clouds because of the strong instability of thin pancake-like condensations (Doroshkevich 1980; Vishniac 1983) and, so, to the formation of complex internal structure of clouds.

These results indicate also that when the process of structure formation is described by the function $F(\boldsymbol{q})$ the ellipsoidal or, in the case of two dimensional problem, elliptical volumes/areas are preferable.

\subsection{Simple approximation}

When only two spherical harmonics are taken into account, the general deformation of any cloud can be described by its deformations along the three orthogonal principal axes, namely, $x_{1}, x_{2}$, and $x_{3}$. In this case, we can use a simpler approach and consider again the three Eqs. (10) instead of (12). With this approach it is possible to obtain some approximate characteristics of the LSS. However, its abilities are restricted and some important problems can be solved only with the general approach (12).

The numbering of principal axes is arbitrary but, further on, we will usually assume that

$\Delta S_{1} / q_{1} \geq \Delta S_{2} / q_{2} \geq \Delta S_{3} / q_{3}$,

and the cloud collapses fastest along the first axis whereas slower collapse - or even expansion takes place along the third axis. For $q_{i} \rightarrow 0$ this choice agrees with the ordering of principal axes of the deformation tensor (Zel'dovich 1970; Shandarin \& Zel'dovich 1989).

The correlations of differences of displacements along the principal axes are relatively small (DD99),

$r_{i j}=\frac{3\left\langle\Delta S_{i}\left(q_{i}\right) \Delta S_{j}\left(q_{j}\right)\right\rangle}{\sigma_{s}^{2} \sigma_{q}\left(q_{i}\right) \sigma_{q}\left(q_{j}\right)} \leq \frac{1}{3}, \quad i \neq j$,

what allows us to consider, in many problems, the components of the deformation field as uncorrelated. Dispersions $\sigma_{s}$ and $\sigma_{q}$ were defined in Eqs. (7) and (11).

For quantitative estimates it is convenient to rewrite Eq. (10) in a dimensionless form

$\Delta r_{i}=\frac{l_{v} q_{i}}{1+z}\left[1-\frac{\tau(z) s_{i}}{\mu\left(q_{i}\right)}\right], \quad s_{i}=\frac{\sqrt{3} \Delta S_{i}}{\sigma_{q} \sigma_{s}}$,

$\mu(q)=\frac{q_{i}}{\sigma_{q}}=\frac{q_{i}}{\sqrt{2\left[1-\xi_{v}\left(q_{i}\right)\right]}}, \quad \eta\left(q_{i}, z\right)=\frac{\mu\left(q_{i}\right)}{\sqrt{2} \tau(z)}$

The probability distribution function (PDF) of dimensionless differences of displacements $s_{i}$ is Gaussian and it is given by:

$\frac{\mathrm{d}^{3} W\left(s_{1}, s_{2}, s_{3}\right)}{\mathrm{d} s_{1} \mathrm{~d} s_{2} \mathrm{~d} s_{3}}=6(2 \pi)^{-3 / 2} \exp \left[-0.5\left(s_{1}^{2}+s_{2}^{2}+s_{3}^{2}\right)\right]$.

Here the factor 6 appears because of the imposed restrictions (15) on the range of variables.

As is seen from Eqs. (8) and (18), for two limiting cases $q_{i} \gg q_{0}$ and $q_{i} \ll q_{0}$, we have

$\mu(q) \approx \frac{\sqrt{q}}{2}, \quad \eta=\frac{1}{2 \tau} \sqrt{\frac{q}{2}}, \quad q_{0} \ll q \ll 1$,

$\mu(q)=\mu_{0} \approx \sqrt{\frac{q_{0}}{3}}, \eta=\eta_{0}=\frac{1}{\tau} \sqrt{\frac{q_{0}}{6}}, q \ll q_{0} \ll 1$,

and the two particles under consideration moving along the $i$ th axis will cross when $s_{i} \geq \sqrt{2} \eta_{i}$. For smaller $q_{i} \leq q_{0} \ll 1$ the condition of the crossing does not depend on the size of the pancake, it means that collapse of all small pancakes takes the same amount of time.

The parameter $q_{0}(4,9)$, characterizes the damping scale in the initial power spectrum and discriminates regions of strong and small correlations of the initial density and velocity fields. 
As is seen from Eq. (21), the condition $\eta_{0}=1$ introduces also the typical redshift, $z=z_{r}$,

$\tau\left(z_{r}\right)=\tau_{r}=\sqrt{\frac{q_{0}}{6}}, \quad 1+z_{r} \approx \frac{\tau_{0}}{\tau_{r}}\left(\frac{1+1.2 \Omega_{\mathrm{m}}}{2.2 \Omega_{\mathrm{m}}}\right)^{1 / 3}$.

At larger redshifts, $z \geq z_{r}, \tau \leq \tau_{r}$, the formation and evolution of the LSS elements strongly depends on the small scale correlations, while later on their influence progressively decrease. For $\Omega_{\mathrm{m}}=0.3, q_{0}=10^{-2}$ and $\tau_{0} \sim 0.3$ we have $z_{r} \approx 6.5$ what coincides with the observed low limit of the redshift of reionization of the Universe (Fan et al. 2001).

\section{Fraction of matter accumulated by structure elements}

The fractions of matter accumulated by pancakes and filaments were discussed in DD99. Here we extend this approach and introduce three cumulative distribution functions for more detailed description of fractions of matter accumulated by pancakes, filaments and walls with different sizes and rates of expansion and/or compression. In turn, this extension allows one to obtain the approximate mass functions of all three kinds of LSS elements and to take partly into account the further evolution of pancakes after their formation. These problems are discussed in Sects. 5 and 7.

We note in Introduction that, in comparison with numerical simulations, our approach decreases the matter fraction assigned to filaments and clouds. Moreover, it does not describe the evolution of clouds within the filaments and pancakes. This means that these estimates correctly approximate matter concentration within walls and pancakes at all redshifts but shift the moment of formation of filaments and clouds to smaller redshifts. Estimates of clouds characteristics are distorted more strongly than those for filaments.

\subsection{Cumulative distribution functions of displacement differences}

As is seen from Eq. (18), the conditions $\Delta r_{1} \leq 0$ and $s_{1} / \sqrt{2} \geq$ $\eta\left(q_{1}\right)=\eta_{1}$ are equivalent and both define collapse of a cloud along the axis of the most rapid compression. To discriminate between pancakes, filaments and walls, we have to use also conditions which restrict the motion along two other directions. For more detailed description of pancakes we have also to restrict the rate of pancake expansion. These conditions are consistent with restrictions (15) and they can be summarized as follows:

$\frac{s_{1}}{\sqrt{2}} \geq \zeta_{1}, \quad \zeta_{1} \geq \zeta_{2} \geq \frac{s_{2}}{\sqrt{2}} \geq \frac{s_{3}}{\sqrt{2}}, \quad \zeta_{3} \geq \frac{s_{3}}{\sqrt{2}} \geq-\infty$,

where the parameters $\zeta_{i}$ restrict the rate of deformation in $i$ th direction.

The first condition in Eq. (23) assumes that for a given $\tau(z)$ the clouds with $\zeta_{1}=\eta_{1} \geq \eta_{0}, \mu\left(q_{1}\right) \geq \sqrt{2} \zeta_{1} \tau$ collapse along the first axis. For $\zeta_{2}=\eta\left(q_{2}\right) \geq \eta_{0}, \zeta_{3}=\eta\left(q_{3}\right) \geq \eta_{0}$, the next two conditions exclude from the consideration collapsed filaments with $\mu\left(q_{2}\right) \geq \sqrt{2} \zeta_{2} \tau$ and clouds with $\mu\left(q_{3}\right) \geq \sqrt{2} \zeta_{3} \tau$. For $\zeta_{3} \leq$ $\zeta_{2} \leq 0$ they restrict the expansion rate of expanding pancakes.
Similar conditions, namely,

$$
\begin{aligned}
& \frac{s_{1}}{\sqrt{2}} \geq \zeta_{1}, \quad \frac{s_{1}}{\sqrt{2}} \geq \frac{s_{2}}{\sqrt{2}} \geq \zeta_{2} \geq \zeta_{3} \geq \frac{s_{3}}{\sqrt{2}} \geq-\infty \\
& \frac{s_{1}}{\sqrt{2}} \geq \zeta_{1}, \quad \frac{s_{1}}{\sqrt{2}} \geq \frac{s_{2}}{\sqrt{2}} \geq \zeta_{2}, \quad \frac{s_{2}}{\sqrt{2}} \geq \frac{s_{3}}{\sqrt{2}} \geq \zeta_{3},
\end{aligned}
$$

with $\zeta_{1}=\eta_{1} \geq \zeta_{2}=\eta_{2} \geq \eta_{0}$, restrict the minimal size of filaments and, for $\zeta_{1}=\eta_{1} \geq \zeta_{2}=\eta_{2} \geq \zeta_{3}=\eta_{3} \geq \eta_{0}=\eta(q=$ $0)$, of clouds collapsed at a given $\tau(z)$.

Integration of the PDF (19) under conditions (23-25) results in the cumulative PDFs that characterize the evolution of the LSS elements:

$$
\begin{aligned}
W_{\mathrm{p}}\left(\zeta_{1}, \zeta_{2}, \zeta_{3}\right)= & \left.\frac{3}{8}\left(1-e_{1}\right)\left(1+e_{3}\right)\right)\left(1+2 e_{2}-e_{3}\right), \\
W_{\mathrm{f}}\left(\zeta_{1}, \zeta_{2}, \zeta_{3}\right)= & \frac{3}{8}\left(1-e_{1}\right)\left(1+e_{3}\right)\left(1+e_{1}-2 e_{2}\right), \\
W_{\mathrm{cl}}\left(\zeta_{1}, \zeta_{2}, \zeta_{3}\right)= & \frac{1}{8}\left(1-e_{1}\right)\left[1+e_{1}+e_{1}^{2}-3 e_{3}\right. \\
& \left.+3 e_{3}\left(e_{3}-e_{1}\right)-3\left(e_{2}-e_{3}\right)^{2}\right]
\end{aligned}
$$

where $e_{i}=\operatorname{erf}\left(\zeta_{i}\right)$.

The functions $W_{\mathrm{p}}, W_{\mathrm{f}}$ and $W_{\mathrm{cl}}$ describe the fractions of matter assigned to pancakes, filaments and clouds, respectively, under conditions (23-25). For $\zeta_{2}=\zeta_{3}=\eta_{2}, e_{2}=e_{3}$, expressions (26) and (27) become identical to those obtained in DD99.

\subsection{Maximal fraction of matter accumulated by structure elements}

From the relations (26)-(28) it follows that in the Zel'dovich theory the maximal fractions of matter that can be accumulated by clouds, filaments and pancakes, for $q_{i}=0, \eta_{i}=\eta_{0}=$ $\tau^{-1}(z) \sqrt{q_{0} / 6}$, are

$W_{\mathrm{cl}} \approx \frac{1}{8} \operatorname{erfc}^{3}\left(\eta_{0}\right), \quad W_{\mathrm{f}}=\frac{3}{8} \operatorname{erfc}^{2}\left(\eta_{0}\right)\left(1+\operatorname{erf}\left(\eta_{0}\right)\right)$,

$W_{\mathrm{p}} \approx \frac{3}{8} \operatorname{erfc}\left(\eta_{0}\right)\left(1+\operatorname{erf}\left(\eta_{0}\right)\right)^{2}$

We see that during the early evolutionary stages, when $z \gg$ $z_{r}, \tau \ll \tau_{r}, \eta_{0} \gg 1$, the fractions (29) increase $\propto \exp \left(-\kappa \eta_{0}^{2}\right)$ with $\kappa=1,2,3$ for pancakes, filaments and clouds. During later evolutionary stages, when $z \ll z_{r}, \tau \gg \tau_{r}, \eta_{0} \ll 1$, we get from Eq. (29):

$W_{\mathrm{cl}} \approx 0.125, \quad W_{\mathrm{f}} \approx W_{\mathrm{p}} \approx 0.375$.

In the Zel'dovich theory motion of matter along the three principal directions as described by the Eq. (10) is considered to be independent. It means that the interaction of matter is neglected and therefore in this approach the matter fraction accumulated by filaments and clouds and the rate of collapse are usually underestimated. For small $\tau$, the acceleration of the collapse along the $x_{2}$ and $x_{3}$ axes after the collapse along the $x_{1}$ axis and 


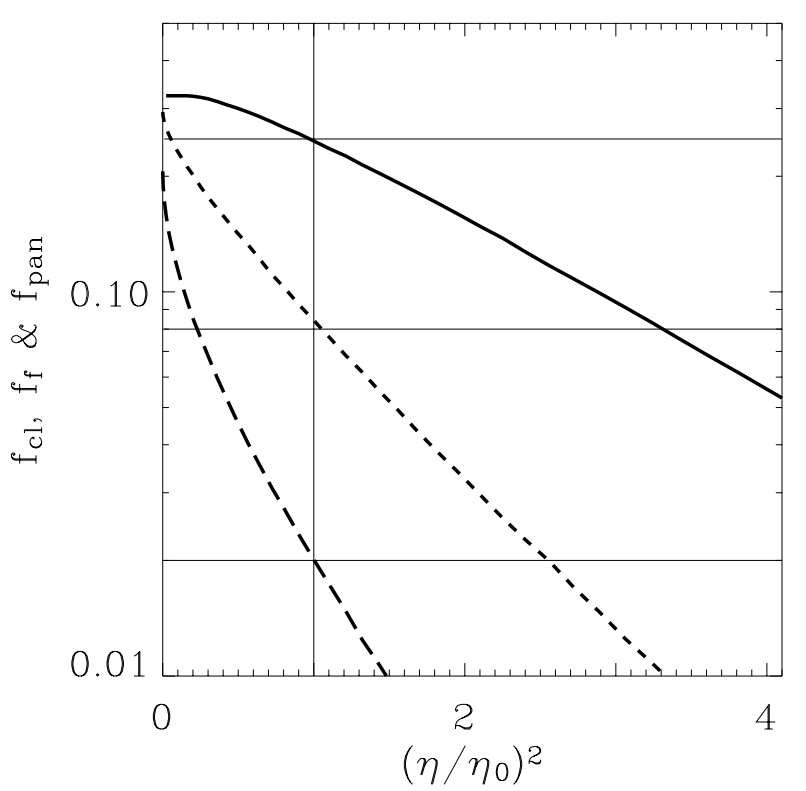

Fig. 1. Fraction of the DM component accumulated by pancakes and walls (solid line), filaments (dashed line) and clouds (long dashed line) plotted versus $\eta^{2}$. These fractions at the moment $\eta=1, \tau=\tau_{r}$, are shown by thin straight lines.

the interaction of small and large scale perturbations increase the fraction of matter accumulated by the collapsing cloud by a factor of about 2 . The same effects increase also the final fraction of matter assigned to filaments and clouds. The popular Press-Schechter approach faces the same problems because the assumption of spherical collapse overestimates the collapse rate and the fraction of collapsed matter.

The cross correlations of orthogonal displacements as given by Eq. (16), though small, increase the maximal fraction of matter accumulated by clouds and filaments up to $W_{\mathrm{cl}} \sim 0.2$ and $W_{\mathrm{f}} \sim 0.3$, respectively, and decrease the fraction of matter accumulated by pancakes to $W_{\mathrm{p}} \sim 0.3$. These maximal fractions are plotted in Fig. 1 versus $\eta^{2} / \eta_{0}^{2}$ for all three types of structure elements. As it is apparent from Fig. 1, already at $\tau=\tau_{r}, \eta=\eta_{0}, \sim 25 \%, 8 \%$ and $2 \%$ of matter is accumulated by pancakes, filaments and clouds, respectively. At $\tau \leq \tau_{r}$, these mass fractions increase $\propto \exp \left(-\kappa \eta^{2}\right), \kappa=1,2,3$ what coincides with Eq. (29).

These estimates of the mass fractions are quite reasonable for large redshifts, when the formation of structure elements from the dispersed matter dominates. At small redshifts the merging of the LSS elements distorts these estimates and, for example, some fraction of filaments and clouds is incorporated into richer walls. These estimates can be improved by a comparison with high resolution simulations and observations of Ly- $\alpha$ forest at high redshifts.

\subsection{Distribution function of the sizes of structure elements}

The relations (26)-(28) are monotonic functions of $\zeta_{i}$ and, for $\zeta_{i}=\eta_{i} \geq \eta_{0}$, they represent the cumulative three parameters distribution functions of structure elements with respect of their sizes measured by differences of Lagrangian coordinates, $q_{1}, q_{2}, q_{3}$, for particles bounding these LSS elements along the principal directions. This joint PDF for all structure elements is

$N\left(q_{1}, q_{2}, q_{3}\right) \propto \exp \left(-\eta_{1}^{2}-\eta_{2}^{2}-\eta_{3}^{2}\right) \frac{\mathrm{d} \eta_{1}}{\mathrm{~d} q_{1}} \frac{\mathrm{d} \eta_{2}}{\mathrm{~d} q_{2}} \frac{\mathrm{d} \eta_{3}}{\mathrm{~d} q_{3}}$,

$\eta_{0} \leq \eta_{i} \leq \infty, \quad 0 \leq q_{i} \leq \infty$

Similar relation allowing for the correlations (16) can be obtained in the same manner.

The expression (30) does not discriminate filaments and pancakes with respect to their expansion rates described in Eqs. (26) and (27) by negative $\zeta_{2}$ and $\zeta_{3}$. To allow such discrimination it is necessary to link $\zeta_{2} \leq 0$ and $\zeta_{3} \leq 0$ with the size of structure elements. The symmetry of the basic characteristics of structure elements with respect to positive and negative $\Delta S_{i}$ discussed in Sect. 2.2 and the relations (11) indicate that such an extension can be achieved by imposing the following limits in Eq. (30):

$\begin{array}{lrr}0 \leq q_{1} \leq \infty, & -\infty \leq q_{2} \leq \infty, & -\infty \leq q_{3} \leq \infty, \\ 0 \leq q_{1} \leq \infty, & 0 \leq q_{2} \leq \infty, & -\infty \leq q_{3} \leq \infty, \\ 0 \leq q_{1} \leq \infty, & 0 \leq q_{2} \leq \infty, & 0 \leq q_{3} \leq \infty,\end{array}$

for pancakes, filaments and clouds, respectively. Here negative $q_{2}, q_{3}$ are associated with expanding filaments and pancakes.

\section{Interaction of small and large scale perturbations. Large scale bias}

High resolution simulations show that at high redshifts a substantial fraction high density halos is accumulated by filaments. In observed redshift surveys galaxies are mainly found within filaments and walls and the population of isolated galaxies is quite small. This strong galaxy concentration within the LSS elements as compared with more homogeneous distribution of baryons and dark matter - large scale bias - can be naturally explained by the interaction of small and large scale perturbations responsible for the formation of galaxies, filaments and walls, respectively. Of course, such bias is enhanced by the acceleration of evolution after formation of filaments and walls.

The spatial modulation of the rate of matter collapse is not described with the approach discussed above because this problem requires the use of at least two point distribution function. This function was introduced in DD99. Here we return to this problem and propose a simple quantitative measure that illustrates effects of this interaction.

\subsection{Simple model of the interaction}

Let us consider the formation of a small pancake within a large scale perturbation with small amplitude. Following Sect. 2.1, we will describe the pancake formation by Eq. (10) with the displacement

$\Delta S_{i}\left(q_{i}\right)=\Delta S_{i}^{\mathrm{sm}}\left(q_{i}\right)+\Delta S_{i}^{\mathrm{ls}}\left(q_{i}\right)$ 
where $\Delta S_{i}^{\mathrm{sm}}\left(q_{i}\right)$ describes the formation of small pancake while $\Delta S_{i}^{\mathrm{ls}}\left(q_{i}\right)$ is related to the large scale perturbation. For simplicity, we assume that the large scale displacement can be taken as a linear function of coordinates

$\Delta S_{i}^{\mathrm{ls}}\left(q_{i}\right) \approx \alpha_{i} \sigma_{s} q_{i}$,

where $\alpha_{i} \geq 0$ and $\alpha_{i} \leq 0$ for the compressed or expanded large scale perturbation. In this case, for the moment of small pancake formation, $\tau_{\mathrm{c}}^{\mathrm{sm}}\left(z_{2}\right)$, we get

$\tau_{\mathrm{c}}^{\mathrm{sm}}\left(z_{2}\right)=\tau_{\mathrm{c}}^{\mathrm{sm}}\left(z_{1}\right)\left[1-\alpha_{1} \sigma_{s} B\left(z_{2}\right)\right]$,

where $\tau_{\mathrm{c}}^{\mathrm{sm}}\left(z_{1}\right)$ is the unperturbed moment of small scale pancake formation, i.e. for $\alpha_{1}=0$. This expression shows that $\tau_{\mathrm{c}}^{\mathrm{sm}}\left(z_{2}\right) \leq \tau_{\mathrm{c}}^{\mathrm{sm}}\left(z_{1}\right)$ and $z_{2} \geq z_{1}$, for $\alpha_{1} \geq 0$, and vise versa. For rough quantitative estimates at $1 \ll 1+z_{3} \leq 1+z_{1}$ and $\tau(z) \propto(1+z)^{-1}$ we can take approximately

$\alpha_{1} \sigma_{s} B\left(z_{\mathrm{c}}\right) \approx \tau_{\mathrm{c}}^{\mathrm{sm}}\left(z_{2}\right) / \tau_{\mathrm{c}}^{\mathrm{ls}}\left(z_{3}\right) \approx\left(1+z_{3}\right) /\left(1+z_{2}\right) \leq 1$,

where the last ratio characterizes the relative amplitude of large and small scale perturbations. In this case we get

$z_{2} \approx z_{1}+1+z_{3} \geq z_{1}, \quad z_{2} \approx z_{1}-1-z_{3} \leq z_{1}$,

for positive and negative $\alpha_{1}$, respectively. This result demonstrates clearly the strong interaction of small and large scale perturbations according to the Zel'dovich theory.

Owing to the linearity of Eq. (32), this result is immediately generalized for a set of large scale perturbations. Thus, for the set of successive merging of earlier formed object we need to substitute $\alpha$ by $\sum_{k} \alpha_{k}$ in Eq. (34) and $z_{3}$ by $\sum_{k} z_{k}$ in Eq. (35). The expression (35) is valid for $z \gg 1$ while the more general expression (34) can be used for all redshifts.

The analysis of numerical simulations indicates that the early formed halos are later on accumulated by filaments and richer walls and, so, are subjected to two, three and more successive compressions. As the structure formation is a deterministic process (with random initial perturbations), all these future mergings accelerate the formation of the first halos already at high redshifts and determine their location within richer walls or filaments at small redshifts. In the opposite case of halo formation within an expanded region we will have deceleration of the collapse and decrease in richness of the halo.

\subsection{Statistical description of the interaction}

This interaction can also be described using the two point correlation function of pancakes introduced in DD99. To do this, we compare the process of formation of two identical pancakes with a surface density $m_{1}=\langle\rho\rangle q_{1}$, and $\mu_{1}=\mu\left(q_{1}\right)$. We assume that the first - a reference - pancake is formed at the "time" $\tau_{1}\left(z_{1}\right)$ and is characterized by the function

$\eta\left(q_{1}, z_{1}\right)=\frac{\mu\left(q_{1}\right)}{\sqrt{2} \tau_{1}}=\left(1+z_{1}\right) \frac{\mu\left(q_{1}\right)}{\sqrt{2} \tau_{0}}$,

as given by Eqs. (18) and (21). For comparison, let us consider the process of formation of the second pancake with the same size, $q_{2}=q_{1}, \mu\left(q_{2}\right)=\mu_{2}=\mu_{1}$, at redshift $z_{2}$ and the successive formation, in the same region, of a pancake with $m_{3} \gg m_{2}$, $q_{3} \gg q_{2}, \mu\left(q_{3}\right)=\mu_{3} \gg \mu_{2}$ at redshift $z=z_{3} \leq z_{2}$, and at the "time" $\tau_{3}\left(z_{3}\right) \geq \tau_{2}\left(z_{2}\right)$. We assume that the larger pancake with the size $q_{3}$ accumulates the earlier formed smaller pancake with the size $q_{2} \ll q_{3}$.

In this case, the interaction of pancakes can be described by replacing $\eta_{2}$ in Eqs. (26) and (30) with the effective parameter $\eta_{\text {eff }}$ defined by the relation (DD99):

$\eta_{\mathrm{eff}}=\left(\eta_{2}-r_{23} \eta_{3}\right) / \sqrt{1-r_{23}^{2}}$,

$r_{23} \approx \frac{2 \mu_{2} \mu_{3}}{q_{2} q_{3}}\left[\xi_{v}\left(\frac{q_{2}-q_{3}}{2}\right)-\xi_{v}\left(\frac{q_{2}+q_{3}}{2}\right)\right]$.

If both pancakes are small, $1 \gg q_{0} \geq q_{3} \gg q_{2}$, then $r_{23} \rightarrow 1$, and the strong small scale correlation of density field decreases the survival probability of smaller pancakes and both pancakes are actually formed at similar redshifts $z_{2} \approx z_{3}$. However, later formation of a larger pancake with $1 \gg q_{3} \geq q_{0}, q_{3} \gg$ $q_{2}, \mu_{3} \gg \mu_{2}$, accelerates compression of the smaller pancake, it increases the effective amplitude of perturbations and shifts formation of pancakes to larger redshifts. For $r_{23} \approx \mu_{2} / \mu_{3} \ll 1$, and at larger redshifts, when $\eta_{i} \propto 1+z$, the general expression (36) can be rewritten more transparently as follows:

$\eta_{\text {eff }} \approx \eta_{2}\left(1-\frac{1+z_{3}}{1+z_{2}}\right)=\frac{\mu\left(q_{2}\right)}{\sqrt{2} \tau_{0}}\left(z_{2}-z_{3}\right)$.

Statistical characteristics of pancakes formed at $z=z_{1}$ and $z=z_{2}$ will be the same for the same value of

$\eta_{\mathrm{eff}}=\eta_{1}=\left(1+z_{1}\right) \frac{\mu\left(q_{1}\right)}{\sqrt{2} \tau_{0}}$.

However, as is seen from Eq. (37), this occurs already at the redshift

$z_{2}=z_{1}+1+z_{3} \geq z_{1}+1$.

This relation gives the same quantitative estimate of the influence of interaction between the large and small scale perturbations as Eq. (35), which increases the actual redshift of pancake formation. Pancakes with the same size $q_{1}$ are now formed earlier at redshift $z_{2} \geq z_{1}$, instead of at $z_{1}$.

The suppression of pancake formation within expanding regions can be considered in the same manner. Thus, for example, for negative $\Delta S_{i}$ and $-s_{i} \geq \sqrt{2} \eta\left(q_{i}, z\right)$ we have instead of Eq. (37)

$\eta_{\mathrm{eff}} \approx \eta_{2}\left(1+\frac{1+z_{3}}{1+z_{2}}\right)=\frac{\mu\left(q_{2}\right)}{\sqrt{2} \tau_{0}}\left(2+z_{2}+z_{3}\right)$,

and from the condition $\eta_{\text {eff }}=\eta_{1}$ we get

$z_{2}=z_{1}-1-z_{3} \leq z_{1}-1$,

what is again identical to Eq. (35) and illustrates how the process of successive formation of pancakes is influencing the moment of their formation.

The influence of large scale perturbations on the process of formation of small scale objects can be considered as the 
manifestation of large scale bias. Fraction of matter accumulated within high density clouds increases rapidly with time (see Fig. 1) and halos formed at larger $z$ contain only a small fraction of mass of halos formed at smaller $z$. But this bias increases the redshift of subclouds formation and their densities, promotes the transformation of DM clouds into observed galaxies, and makes the internal structure of clouds more complex. Regular distribution of young galaxies within high density filaments suppresses their feedback and fosters further formation of galaxies.

\subsection{Comparison with simulations}

We compare these expectations with the high resolution simulation (Klypin et al. 1999; Schmalzing et al. 1999) performed with adaptive code in a box of $\left(60 h^{-1} \mathrm{Mpc}\right)^{3}$ with $N_{\text {tot }}=(256)^{3}$ particles for the Harrison-Zel'dovich primordial power spectrum and the BBKS transfer function. This box size and small scale cutoff of the power spectrum approximately correspond to $q_{0} \sim 10^{-2}, M_{\mathrm{DM}} \sim 1 \mathrm{keV}$.

To test the impact of environment on the properties of high density clouds we use the Minimal Spanning Tree technique described in Doroshkevich et al. (2001, 2004). For a given threshold linking length or for the threshold overdensity above the mean density, this technique allows one to select all clouds from the sample without any restrictions of their shape. For each selected cloud its richness is also easily defined.

The matter distribution at redshifts $z=4, z=1$ and $z=0$ were analyzed. As the first step, the original samples were decomposed into subsamples of high and low density regions (HDRs and LDRs). The subsamples of high density regions accumulate $\sim 40-45 \%$ of all DM particles within richer clouds selected for the threshold density equal to the mean density. Subsamples of LDRs were prepared by removing the HDRs from the full samples.

At the second step, the high density structure elements were selected within HDRs and LDRs with a set of the threshold linking lengths. The matter fraction accumulated by the elements is plotted in Fig. 2 versus the threshold overdensity used for the selection. As is seen from this Figure, at all redshifts the majority of highly compressed matter is situated within the HDRs. This excess is moderate at $z=0$ and progressively increases with redshift. Special test shows that the high density clouds selected at $z=4$ are equally distributed between HDRs and LDRs selected at $z=0$. This fact is consistent with almost equal concentration of galaxies within the HDRs and LDRs at $z=0$ in the observed surveys. It indicates also that the efficiency of the interaction of small and large scale perturbations increases with redshift.

Impact of environment on the properties of galaxy groups selected within HDRs and LDRs in the observed SDSS DR1 survey are discussed in Doroshkevich et al. (2004). As before, properties of such groups selected within HDRs and LDRs are found to be strongly different. Similar correlation of properties of simulated high density halos with their environment and their evolutionary histories was also found in

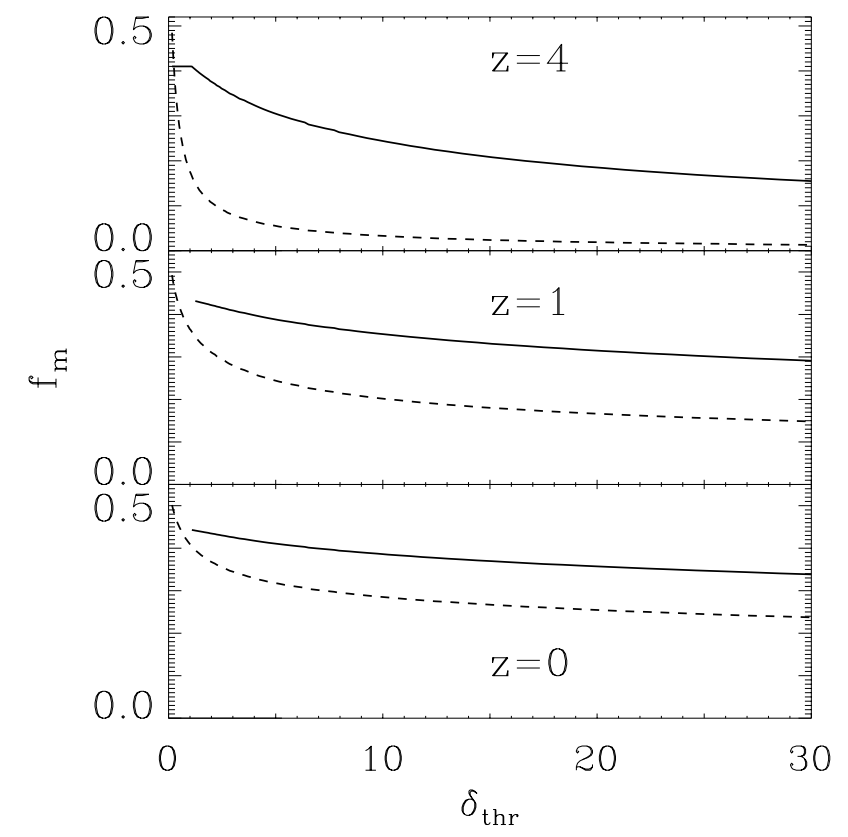

Fig. 2. Fractions of matter, $f_{\mathrm{m}}$, accumulated by structure elements situated within HDRs (solid lines) and LDRs (dashed lines) are plotted vs. the threshold overdensity for simulated DM distribution at three redshifts.

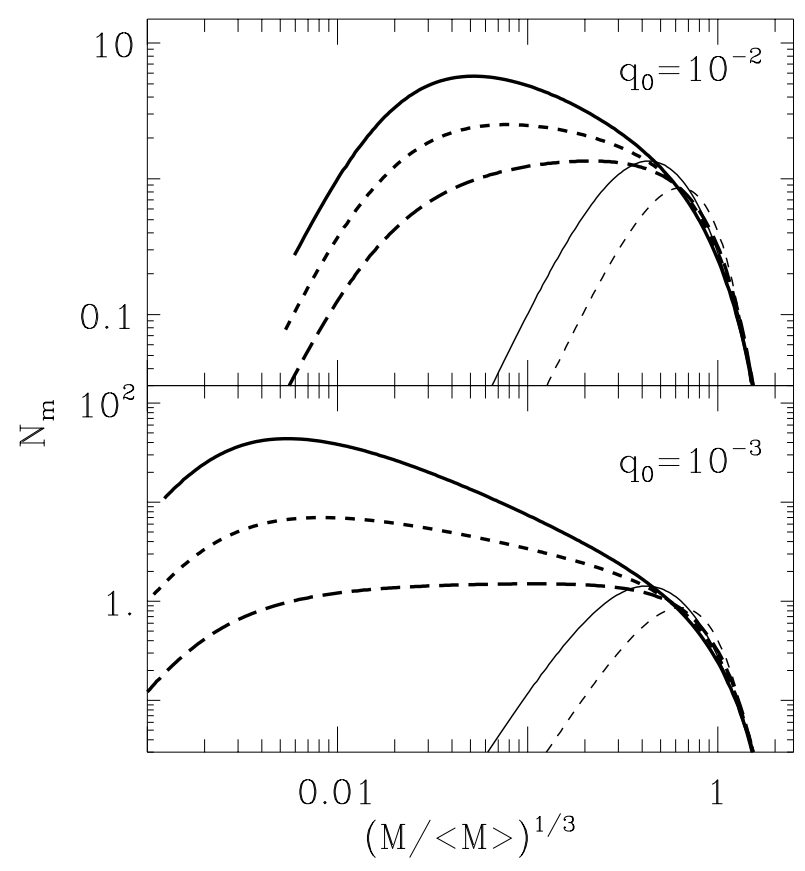

Fig. 3. Functions $N_{\mathrm{w}}$ (thick solid line), $N_{\mathrm{f}}$ (thick dashed line) and $N_{\mathrm{c}}$ (thick long dashed line) for two values of $q_{0}$ plotted vs. $(M /\langle M\rangle)^{1 / 3}$ for $\tau=0.3$. For $\tau \approx \tau_{r}=\sqrt{q_{0} / 6}$ and $\tau \approx 0.3 \tau_{r}$, functions $N_{\mathrm{w}}$ are plotted by thin solid and dashed lines, respectively.

Gottlöber et al. (2001, 2002, 2003) where different statistical method - the mark correlation function - was used. 


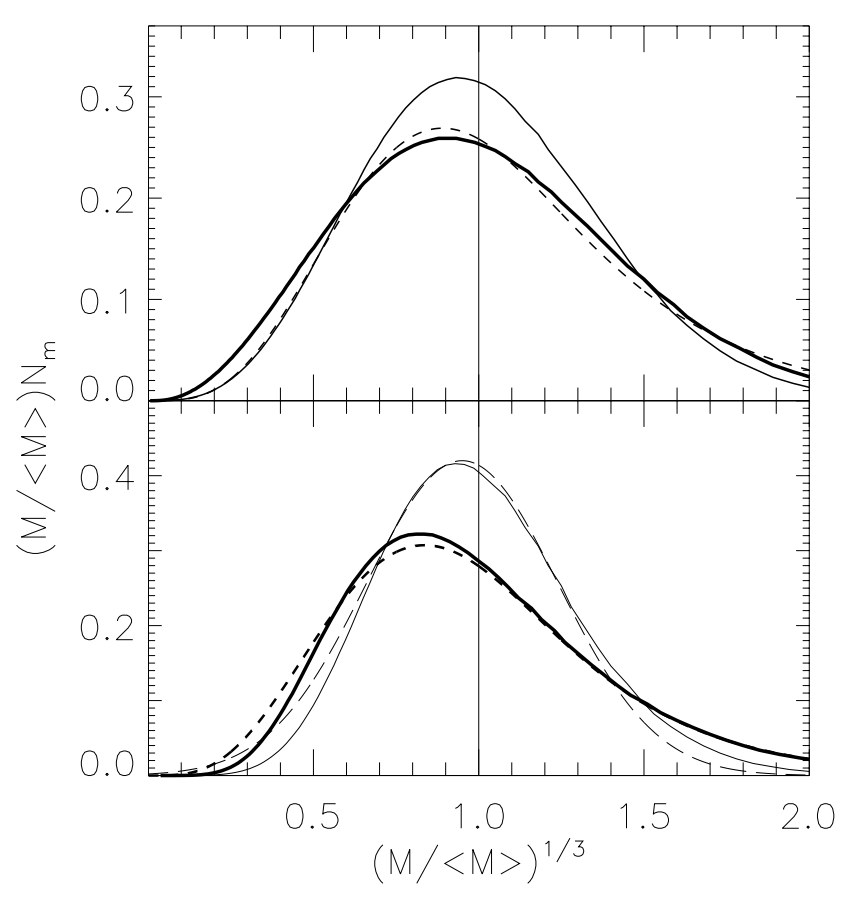

Fig. 4. Top panel: functions $M /\langle M\rangle N_{\mathrm{w}}$ (thick solid line) and $M /\langle M\rangle N_{\mathrm{c}}$ (thin solid line) plotted vs. $(M /\langle M\rangle)^{1 / 3}$ for $\tau=0.3$. Fit (47) is drawn by dashed line. Bottom panel: functions $M /\langle M\rangle N_{\mathrm{w}}$ for $\tau \approx \tau_{r}=\sqrt{q_{0} / 6}$ (thick solid line) and $\tau \approx 0.3 \tau_{r}$ (thin solid line) plotted vs. $(M /\langle M\rangle)^{1 / 3}$. Fits (45) and (46) are drawn by thin dashed and long dashed lines, respectively.

\section{Mass function and rotation of structure elements}

The popular Press-Schechter formalism focuses main attention on the spherical collapse and the achieved final critical overdensity (see, e.g., Press \& Schechter 1974; Gunn \& Gott 1974; Epstein 1983; for review in Loeb \& Barkana 2001). It can be extended for elliptical clouds (see, e.g., Sheth \& Tormen 2002). In spite of so strong restrictions on the process of collapse it successfully describes the simulated mass distribution of high density halos. In Zel'dovich theory of gravitational instability similar approach allows one to find the mass function of all high density structure elements - clouds, filaments, and walls or pancakes - without any assumptions about their initial and final shapes, stability, relaxation and achieved overdensity. In this regard, our approach is much more general then the PressSchechter formalism.

The close connection of the observed objects with the initial power spectrum and the generic origin of galaxies and other observed elements of the LSS is clearly seen from the expression (30) which applies to all structure elements. Due to symmetry of the relation (30) with respect to variables $q_{1}, q_{2}$ and $q_{3}$ and because the mass functions are normalized, the restrictions (23)-(25), used for the determination of the mass fractions of different structure elements, are now of no importance and they lead only to renumeration of coordinates. Thus, in the Zel'dovich approach, the mass functions of the various LSS elements differ only by their corresponding survival probability (see, e.g., Peacock \& Heavens 1990; Bond et al. 1991).

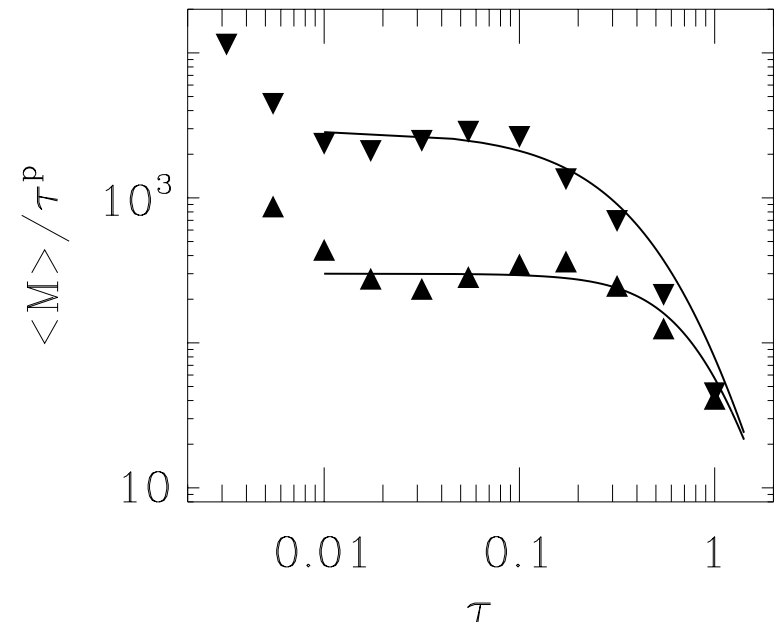

Fig. 5. Functions $\langle M\rangle / \tau^{4.3}$ (triangles, $q_{0}=10^{-2}$ ) and $\langle M\rangle / \tau^{5.2}$ (up down triangles, $q_{0}=10^{-3}$ ) plotted vs. $\tau$. Fits (50) and (51) are drawn by solid lines, respectively.

Our approach describes correctly the formation of pancakes and walls but, as compared with simulations, it decelerates the formation of filaments and clouds and decreases their mean masses for a given redshift. Moreover, the evolution of high density clouds embedded within filaments and walls is accelerated due to the impact of environment and, perhaps, is better described by the coagulation approach. Indeed, as is noted in Sect. 5.7, at $z=4$ the mass functions of filaments and clouds are found to be quite similar to the expected ones. However, at $z=0$ these functions can also be fitted by power laws what demonstrates a possible impact of coagulation processes. This problem requires further extended investigation similar to that performed in Gottlöber et al. (2001, 2002, 2003) for high density halos.

\subsection{Initial shape of collapsed clouds}

To find the mass function of structure elements we rewrite the general relation (30) in spherical coordinates

$q_{1}=R \cos \theta, \quad q_{2}=R \sin \theta \cos \phi, \quad q_{3}=R \sin \theta \sin \phi$,

where $0 \leq R \leq \infty$ and $0 \leq \theta, \phi \leq \pi / 2$ characterize the mass and shape of the collapsed structure elements in the Lagrangian coordinates:

$M \propto R^{3}, \quad \operatorname{tg} \phi=q_{3} / q_{2}, \quad \operatorname{tg} \theta=\sqrt{q_{2}^{2}+q_{3}^{2}} / q_{1}$.

For spherical clouds $\operatorname{tg} \phi=1, \phi=\pi / 4, \operatorname{tg} \theta=\sqrt{2}$. As was noted above, here we will neglect the restrictions (23)-(25) and differences in limits of $q_{i}$ (31).

This result demonstrates that in the Zel'dovich approximation the distribution of shapes of initial clouds described by the parameters $\theta$ and $\phi$ in Eq. (39) is continuous and the final properties of structure elements, such as their shape, energy and overdensity, depend mainly upon the velocity or deformation field within clouds. In particular, even high density compact relaxed halos can be formed through the collapse of initially asymmetrical clouds. Some information about the initial shape 


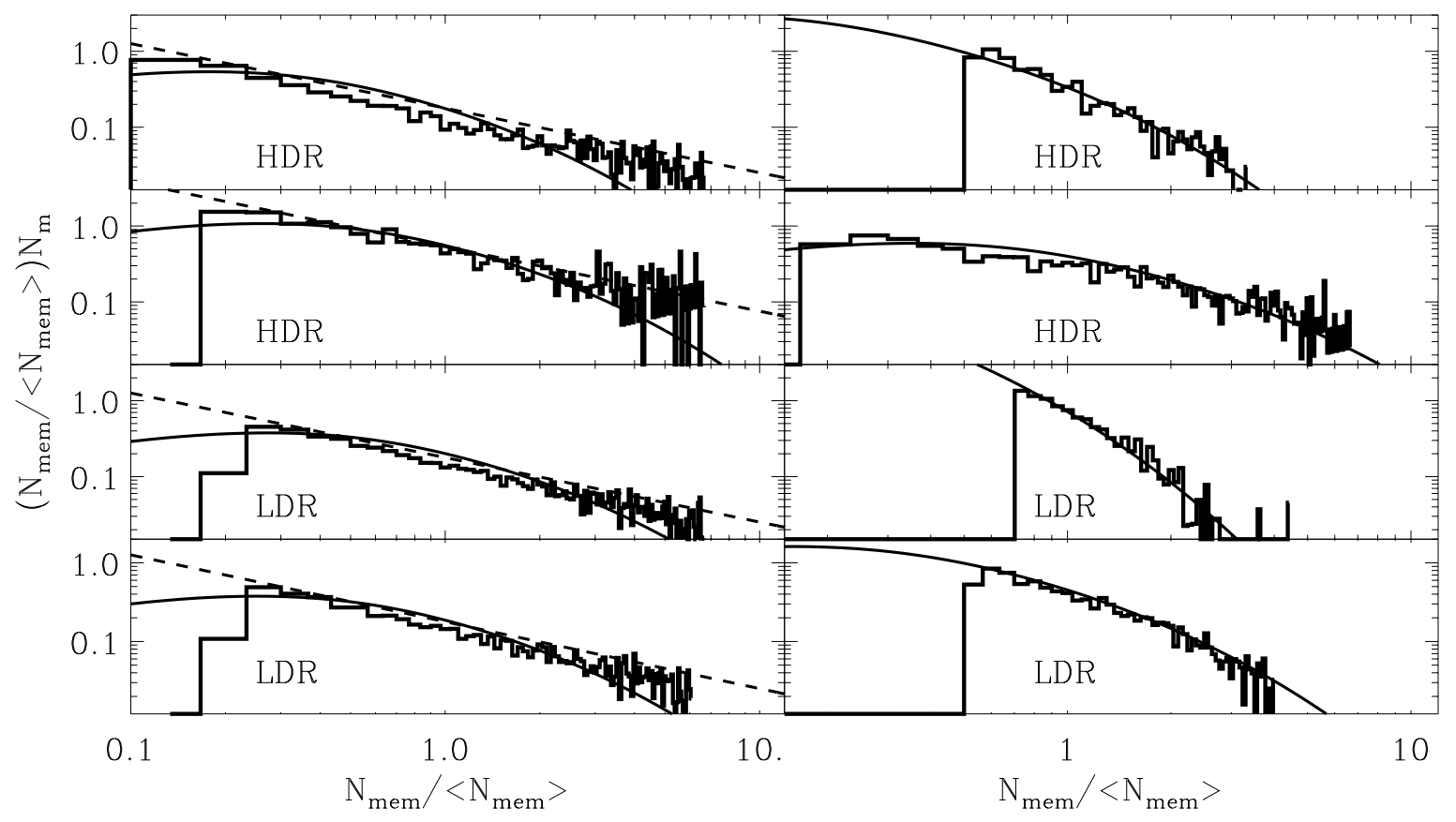

Fig. 6. Mass functions of galaxy clouds, $\left.N_{\mathrm{mem}} /\left\langle N_{\mathrm{mem}}\right\rangle\right) N_{m}$, selected at redshifts $z=0$ (left panels) and $z=4$ (right panels) in HDRs and LDRs for two threshold linking lengths. Fits (47) and (56) are plotted by solid and dashed lines, respectively.

of collapsed clouds is retained in the angular momentum of observed clouds discussed below.

On the other hand, for all clouds, the first step of collapse is the formation of a pancake-like objects (Zel'dovich 1970; Shandarin et al. 1995) that are unstable and will rapidly break up into a system of low mass subclouds (Doroshkevich 1980; Vishniac 1983). This instability stimulates formation of numerous satellites of the largest central object and makes it difficult to observationally distinguish between isolated galaxies and these satellites.

For the more massive clouds with $1>q_{i} \gg q_{0}, \eta_{i} \propto \sqrt{q_{i}}$, we have from Eq. (30):

$\mathrm{d}^{3} W=\frac{\exp \left(-R \psi(\theta, \phi) / 8 \tau^{2}\right)}{\sqrt{R^{3} \cos \theta \sin 2 \phi}} \frac{\mathrm{d} R^{3} \mathrm{~d} \phi \mathrm{d} \theta}{6 \pi^{3 / 2} \tau^{3}}$,

$\psi(\theta, \phi)=\cos \theta+\sqrt{2} \sin \theta \cos (\phi-\pi / 4)$.

This relation shows that the fraction of initially massive spherical clouds with $\psi=\sqrt{3}$ is exponentially suppressed and asymmetric massive clouds dominate.

For low mass clouds with $q_{i}<q_{0}$, we get for the mass function

$\eta_{i} \approx \frac{1}{2 \tau} \sqrt{\frac{q_{0}}{3}}\left(1+\frac{q_{i}^{2}}{6 q_{0}^{2}}+\ldots\right)$,

$\mathrm{d}^{3} W \propto R^{3} \mathrm{~d} R^{3} \tau^{-3} \sin 2 \theta(1-\cos 2 \theta) \sin 2 \phi \mathrm{d} \theta \mathrm{d} \phi$,

and the formation of low mass clouds with $M \leq M_{\rho}$ (6) is suppressed. However, in this case the existence of approximately spherical initial clouds is much more probable.

\subsection{Survival probability of objects}

As it was shown above formation of low mass structure elements is usually suppressed because they could be absorbed by larger objects formed at the same time and in the same region. This process is described by the survival probability of structure elements and it is different for clouds, filaments and walls. As was shown in DD99, for walls formed through the 1D compression along the $q_{1}$ axis the survival probability can be taken as

$W_{\text {surv }}=\operatorname{erf}\left(\sqrt{q_{1} / 8 \tau^{2}}\right)$.

For filaments and clouds formed through the 2D and 3D compression the survival probabilities can be taken as

$W_{\text {surv }}=\operatorname{erf}\left(\sqrt{q_{1} / 8 \tau^{2}}\right) \operatorname{erf}\left(\sqrt{q_{2} / 8 \tau^{2}}\right)$,

$W_{\text {surv }}=\operatorname{erf}\left(\sqrt{q_{1} / 8 \tau^{2}}\right) \operatorname{erf}\left(\sqrt{q_{2} / 8 \tau^{2}}\right) \operatorname{erf}\left(\sqrt{q_{3} / 8 \tau^{2}}\right)$,

respectively. Evidently, so defined survival probability is small for low mass objects and $W_{\text {surv }} \rightarrow 1$ for massive objects.

Integration of the PDF Eq. (40) corrected for the survival probability (42)-(44), $W_{\text {surv }} \mathrm{d}^{3} W$, over angular variables for given $R, q_{0}$ and $\tau$ allows one to find the mass functions of structure elements: clouds, filaments, walls and pancakes.

\subsection{Mass function of structure elements}

The mass function $N_{m}$ and the function $M /\langle M\rangle N_{m}$ are plotted in Figs. 3 and 4 for $q_{0}=10^{-2}$, and $q_{0}=10^{-3}$ and for the three more interesting values of $\tau=0.3, \tau=\tau_{r}$, and $\tau \approx 0.3 \tau_{r}$. 
If $\tau \sim 0.3$ corresponds approximately to the present epoch (see Sect. 7.1), then $\tau \sim \tau_{r}$ (see Eq. (22)) and $\tau \sim 0.3 \tau_{r}$ describe the early period of structure formation, when the influence of small scale correlations of initial density and velocity fields is more important.

As is seen from Fig. 3 at $\tau \leq \tau_{r}$ the mass functions for all three kinds of structure elements are identical, what is a direct consequence of strong correlations of small scale perturbations. However, the difference between these mass functions increases with time and at $\tau \approx 0.3$ it becomes significant, especially for $q_{0}=10^{-3}$.

As is seen from comparison of Figs. 3 and 4, in all the cases the numerous low mass structure elements contain only negligible fraction of mass and for all $\tau$ the main mass is concentrated within structure elements with $M \sim(0.2-0.7)\langle M\rangle$. For $\tau<\tau_{r}$ the impact of the parameter $q_{0}$ on the shape of mass functions is negligible and, for both chosen values of $q_{0}$, the functions $M N_{m}(M)$ are well fitted by the Gauss function (45). This result reflects again a strong small scale correlation of the initial density field and, as was discussed in Sect. 5.2, a small survival probability of low mass structure elements.

At $\tau \geq \tau_{r}$ the mass function becomes wider because the continued formation of low mass structure elements is accompanied by progressive mass concentration within massive elements with $M \sim\langle M\rangle \gg M_{\rho}$ (6). As is seen in Fig. 3, during this period the small scale cutoff of initial power spectrum and the parameter $q_{0}$ provide the low mass cutoff of the mass function. For such redshifts, the mass functions for objects with $M \leq\langle M\rangle$ and $M \geq\langle M\rangle$ are described respectively by power and exponential laws.

Disregarding the low mass "tails" of mass functions, we fit the mass function for both values of $q_{0}$ by:

$x^{3} N_{m}(x) \approx 0.4 \exp \left(-(x-0.95)^{2} / 0.17\right), \quad \tau \approx 0.3 \tau_{r}$,

$x^{3} N_{m}(x) \approx 20 x^{2} \exp (-4.1 x) \operatorname{erf}\left(x^{2}\right), \quad \tau=\tau_{r}$,

$x^{3} N_{m}(x) \approx 12.5 x^{2} \exp (-3.7 x) \operatorname{erf}\left(x^{2}\right), \quad \tau=0.3$,

where $x=(M /\langle M\rangle)^{1 / 3}$. These fits are plotted in Fig. 3. For $\tau \approx 1$, we have

$x^{3} N_{m}(x) \approx 8 x^{3 / 2} \exp (-3.1 x) \operatorname{erf}\left(x^{9 / 4}\right)$.

This function can be used to characterize the observed and simulated filaments and walls which are still forming.

By definition, the mass functions must satisfy two normalization conditions

$3 \int_{0}^{\infty} x^{2} N_{m}(x) \mathrm{d} x \approx 3 \int_{0}^{\infty} x^{5} N_{m}(x) \mathrm{d} x \approx 1$.

Fits (45)-(48) better satisfy the second condition (49) while the first one is violated more strongly. These mass functions are similar to the expression (40) and $N_{m} \propto \exp (-x)$, for $x \gg 1$ and $\tau \geq \tau_{r}$.

\subsection{Mean mass of objects}

The mean mass of objects is quite sensitive to the coherent length of initial density field. For the same $q_{0}$ as above, the mean mass of clouds is plotted in Fig. 5 versus $\tau$ together with fits

$\langle M\rangle \approx \frac{3 \times 10^{2} \tau^{4.3}}{\left(1+\tau^{2}\right)^{2.4}} M_{v}, \quad$ for $\quad q_{0}=10^{-2}$,

$\langle M\rangle \approx \frac{3 \times 10^{3} \tau^{5.2}}{\left(1+\tau^{1.2}\right)^{5.2}} M_{v}, \quad$ for $\quad q_{0}=10^{-3}$,

which describe quite well the redshift evolution of $\langle M\rangle$ for $\tau \geq$ $\tau_{r}$. For $\tau \leq \tau_{r}$ the much slower evolution of the mean mass is described by

$\langle M\rangle \approx \tau^{3} M_{v}, \quad$ for $\quad q_{0}=10^{-2}$,

$\langle M\rangle \approx 4 \times 10^{-2} \tau^{3} M_{v}$, for $q_{0}=10^{-3}$,

what again emphasizes the impact of the small survival probability of clouds at $\tau \leq \tau_{r}$.

For $\tau \geq \tau_{r}$, the mean masses of pancakes and filaments are smaller than those for clouds by a factor of $\sim 1.5-2$.

\subsection{Comparison with the Press-Schechter formalism}

The mass function $N_{m}$ describes all structure elements clouds, filaments, and walls or pancakes - without assumptions about their shapes and achieved overdensity. However, the relation (48) is quite similar to the Press-Schechter mass function for scale-free power spectra and $k$ corresponding to typical objects, $p \propto k^{-2}$ :

$\frac{M}{\langle M\rangle} N_{\mathrm{PS}} \mathrm{d} \xi=\frac{8}{45 \sqrt{\pi}} \xi^{1 / 6} \exp \left(-\xi^{1 / 3}\right) \mathrm{d} \xi$

$\xi=1.875 M /\langle M\rangle, \quad\langle\xi\rangle=15 / 8$.

In turn, for larger redshifts, $\tau \sim \tau_{r}$, and for a power spectrum with a cutoff at $k \sim k_{m x}$, both approaches predict the suppression of formation of low mass objects with $M \ll\langle M\rangle$ (Loeb \& Barkana 2001). This similarity is quite apparent as both relations are based on the same initial power spectrum. It indicates that the difference between the Press-Schechter and a more general Zel'dovich approach is quantitative rather then qualitative and these approaches are complementary to each other.

\subsection{Impact of coagulation processes}

All mass functions discussed above are related to the process of formation of structure elements and they do not take into account the later nonlinear evolution described by the coagulation equation (Smoluchowski 1916; Silk \& White 1978; Sheth \& Pitman 1997). This is not so important for walls and filaments for which the merging and coagulation are controlled mainly by the initial velocity field. But the non linear evolution can essentially distort the mass function of high density clouds accumulated by richer walls and filaments. 
In contrast with expressions (45)-(48) and (54) the coagulation process leads to the mass function

$N_{\mathrm{c}}(x) \propto x^{-v} \exp (-x), \quad x=M /\langle M\rangle \geq x_{\min }$,

where the power index $v \sim 3 / 2$ depends upon the aggregation rate (see, e.g., Silk \& White 1978). This mass function strongly differs from those discussed above. This means that the shape of the observed and simulated mass functions measures the possible influence of coagulation processes.

\subsection{Comparison with simulations}

The theoretical fits (47) and (55) can be compared with the mass functions found for matter distributions at redshifts $z=4$, and $z=0$ in the high resolution simulation (Klypin et al. 1999; Schmalzing et al. 1999) discussed in Sect. 4.3. As was described in Sect. 4.3, at both redshifts the full samples were divided into subsamples of high and low density regions (HDRs and LDRs) dominated by walls and filaments. The selection of structure elements was also performed with the Minimal Spanning Tree technique described in Doroshkevich et al. (2001, 2004).

The mass functions of high density clouds found for the HDRs and LDRs separately are plotted in Fig. 6. The basic parameters of the same samples of selected clouds are listed in Table 1, where $\delta_{\text {thr }}$ and $\langle\delta\rangle$ are threshold and mean overdensities of clouds above the mean density of the sample, $f_{\text {pnt }}$ is the fraction of points accumulated by clouds, $N_{\mathrm{cl}}$ and $\left\langle N_{\mathrm{mem}}\right\rangle$ are the number and the mean richness of clouds. At both redshifts, very massive structure elements are formed through the percolation process and they cannot be described by the expressions (47), (54) or (55). For this reason, they were excluded from the analysis of the mass distribution. However, these clouds are included in estimates of the matter fraction $f_{\mathrm{pnt}}$ accumulated by structure elements as they represent actual filaments and walls. The difference between both $f_{\mathrm{pnt}}$ and $\left\langle N_{\mathrm{mem}}\right\rangle$ for clouds selected with the same $\delta_{\text {thr }}$ within HDRs and LDRs confirms a significant impact of environment on the properties of clouds (see Sect. 4).

At both redshifts, the samples selected with high $\delta_{\text {thr }}$ represent properties of small fraction of high density clouds, while samples selected with small $\delta_{\text {thr }}$ are formed mainly by unrelaxed filaments and walls. The cutoff of the simulated mass functions at low masses caused by a finite resolution increases the measured mean richness of selected clouds as compared with theoretical expectations (50). Because of this, the mean richness was used as a fit parameter in the relations (47) plotted in Fig. 6. However, the shape of the mass function (47) was not altered. As is seen from Fig. 6, this expression fits well the simulated mass functions for both high density clouds and only partly relaxed filaments and walls.

However, at the redshift $z=0$ the simulated mass functions are well fitted also by a power law

$x N_{m}(x) \propto x^{-0.85}, \quad x=N_{\text {mem }} /\left\langle N_{\text {mem }}\right\rangle$,

similar to Eq. (55). It indicates the possible influence of the coagulation processes on the parameters of selected high density
Table 1. Parameters of structure elements selected in HDRs and LDRs at $z=0$ and 4 .

\begin{tabular}{lcrlrr}
\hline \hline & $\delta_{\mathrm{thr}}$ & $\langle\delta\rangle$ & $f_{\mathrm{pnt}}$ & $N_{\mathrm{cl}}$ & $\left\langle N_{\mathrm{mem}}\right\rangle$ \\
\hline & & & $z=4$ & & \\
HDR & 490 & 3007 & 0.015 & 2187 & 101 \\
HDR & 1.6 & 15 & 0.37 & 5311 & 313 \\
LDR & 7.1 & 139 & 0.004 & 963 & 75 \\
LDR & 1.6 & 15 & 0.036 & 6104 & 108 \\
\hline & & $z=0$ & & & \\
HDR & 86 & 1200 & 0.18 & 11155 & 273 \\
HDR & 22 & 645 & 0.33 & 2908 & 342 \\
LDR & 22 & 712 & 0.21 & 8484 & 270 \\
LDR & 1. & 62 & 0.43 & 9629 & 261
\end{tabular}

The few richest clouds formed through percolation process are excluded from calculations of $\left\langle N_{\text {mem }}\right\rangle$.

clouds and even filaments and walls. At the redshift $z=4$ this influence is weak and the mass functions are evidently exponential.

It is especially important that at both redshifts the relations (47) and (54) successfully reproduce the mass functions of unrelaxed filaments and walls that are far from the spherical shape. This fact demonstrates a moderate influence of the shape of collapsed clouds on their mass and the validity of Zel'dovich approach which considers the dynamical characteristics of collapsed clouds rather than their shape.

Similar analysis of the observed SDSS DR1 sample of galaxies can be found in Doroshkevich et al. (2004).

\subsection{The angular momentum of collapsed clouds}

The tidal interaction of perturbed matter creates both the anisotropy of the collapse and the angular momentum of forming clouds (Hoyle 1949; Peebles 1969; Doroshkevich 1970; White 1984; Bullock et al. 2001; Vivitska et al. 2002). Usually the angular momentum is defined for the high density relaxed clouds and is expressed through the so called $\lambda$-parameter. Here we extend the approach developed in Peebles (1969), Doroshkevich (1970) and White (1984) and express the created angular momentum for structure elements in the framework of statistical approach used in this paper.

For this purpose we use the general Eq. (1) together with the corresponding expression for the velocity of fluid element

$v_{i}=\frac{\mathrm{d} r_{i}}{\mathrm{~d} t}=H(z) r_{i}-\frac{H(z)}{1+z}(\beta(z)-1) B(z) S_{i}$,

where $\beta(z)=(1+z) \mathrm{d} \ln B / \mathrm{d} z, H(z)$ is the Hubble parameter, and $B(z)$ was introduced by Eq. (2). As usual, we define the angular momentum of a particle as

$j_{i}=\epsilon_{i j k} r_{j} v_{k}=J_{0} \epsilon_{i j k} \tilde{q}_{j} S_{k}, \quad J_{0}=\frac{H(z)(\beta-1) B(z)}{(1+z)^{2}}$

where $\epsilon_{i j k}$ is the unit antisymmetric tensor and the function $J_{0}(z)$ describes time variations of the angular momentum. Let us note that the description of the angular momentum through the deformation tensor (see, e.g., White 1984) is useful methodically but cannot be applied to more massive objects because of the small correlation scale of this tensor. 
The angular momentum of a cloud is defined by the integral over the corresponding collapsed volume, $V$,

$\left\langle J^{2}\right\rangle=J_{0}^{2} \frac{\sigma_{s}^{2} l_{v}^{2}}{3 V^{2}} \int_{V} \mathrm{~d}^{3} \tilde{p} \mathrm{~d}^{3} \tilde{q} I(\boldsymbol{p}, \boldsymbol{q})$,

$I(\boldsymbol{p}, \boldsymbol{q})=2(\boldsymbol{p q}) G_{1}(|\boldsymbol{p}-\boldsymbol{q}|)+\left(p^{2} q^{2}-(\boldsymbol{p q})^{2}\right) G_{2}(|\boldsymbol{p}-\boldsymbol{q}|)$,

(the functions $G_{1}$ and $G_{2}$ are introduced in DD99 and Appendix A). It depends upon the size and shape of the collapsed region, statistical characteristics of which are described by Eq. (40). For low mass early formed clouds with $p \leq q_{0}$, $q \leq q_{0}$, we get

$I \approx\left[3(\boldsymbol{p q})^{2}-p^{2} q^{2}\right] / q_{0}$,

and, for example, for ellipsoidal clouds with axes $a_{1}, a_{2}$, and $a_{3}$ we have

$\int_{V} \mathrm{~d}^{3} p \mathrm{~d}^{3} q I \propto \frac{1}{q_{0}}\left[a_{12}^{2}+a_{13}^{2}+a_{23}^{2}\right], \quad a_{i j}=a_{i}^{2}-a_{j}^{2}$,

what is identical with the expression found already in Doroshkevich (1970).

For larger clouds with $p \gg q_{0}, q \gg q_{0}$, we get

$I \approx \frac{5(\boldsymbol{p q})^{2}-p^{2} q^{2}-2(\boldsymbol{p q})\left(p^{2}+q^{2}\right)}{\sqrt{p^{2}+q^{2}-2(\boldsymbol{p q})}}$,

and for spherical clouds $\left\langle J^{2}\right\rangle=0$. Numerical integration of Eq. (59) shows that for large elliptical clouds $\left\langle J^{2}\right\rangle$ depends on the axes $a_{i}$ in a similar way as for small clouds (61).

For the high density halos formed by the successive mergings of subhalos a statistical model was proposed in Vivitska et al. (2002). Comparison with simulations and the current status of the problem can be found in Bullock et al. (2001) and Vivitska et al. (2002).

\section{Statistical characteristics of filaments}

In addition to the fraction of matter accumulated by filaments (27) and the mass function of filaments given by Eqs. (45)-(48), we will consider here also the linear density of galaxies along a filament, $\Sigma_{\text {fil }}$, defined as a mass per unit length of filament, and the mean surface density of filaments, $\sigma_{\text {fil }}$, defined as the mean number of filaments intersecting a unit area of arbitrary orientation. These characteristics are weakly dependent upon the nonlinear evolution of matter compressed within filaments and are mainly defined by properties of the initial velocity field and the process of filaments formation and merging.

The measured characteristics of filaments depend also upon the threshold linking length, $r_{\text {lnk }}$, used for the filament selection, which determines the threshold overdensity bounding the filaments. Now only richer filaments can be selected in both observed and simulated catalogs what restricts their quantitative characteristics. Because of this, here we will only discuss characteristics of richer filaments.

The distribution function of filaments linear density describes their frequency distribution with respect to the amount

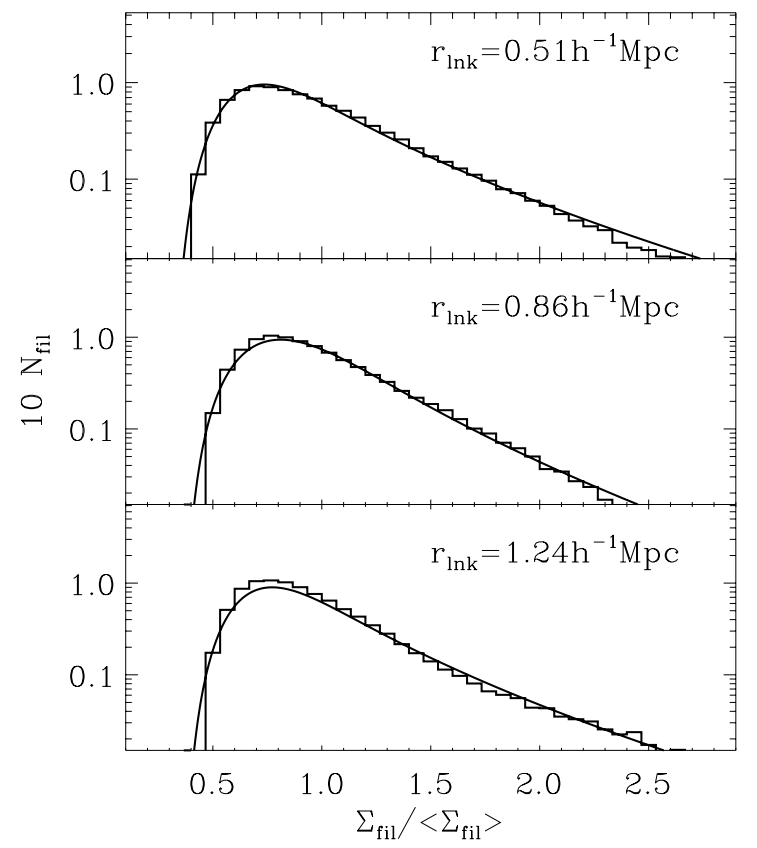

Fig. 7. Distribution function, $N_{\text {fil }}$, for the linear density of DM particles in filaments selected at three linking lengths, $r_{\text {lnk }}$. Fits (64) are plotted by solid lines.

of matter per unit length of filaments. As was discussed in DD99, this function can be obtained by integrating Eq. (30) over all $q_{3}$ and over the ratio $q_{1} / q_{2}$. For $q_{0} \ll 1, q_{0} \ll q \leq 1$ and using the survival probability for low mass filaments given by (43) we have for the PDF of richer filaments with $\Sigma_{\text {fil }} \propto$ $q_{1}^{2}+q_{2}^{2} \geq\left\langle\Sigma_{\mathrm{fil}}\right\rangle$

$N_{\text {fil }} \approx \frac{1.5}{\left\langle\Sigma_{\text {fil }}\right\rangle} \exp \left(-\sqrt{3 \Sigma_{\text {fil }} /\left\langle\Sigma_{\text {fil }}\right\rangle}\right), \quad\left\langle\Sigma_{\text {fil }}\right\rangle \approx 48 \tau^{4}$.

In Fig. 7 the PDFs, $N_{\text {fil }}$, are plotted for filaments selected from two simulated DM distributions at $z=0$ (Jenkins et al. 1998, top panel; Cole et al. 1998, middle and bottom panels) for three linking lengths $r_{\text {lnk }}$. The linear density of matter, $\Sigma_{\text {fil }}$, was measured by the ratio of the number of points and the length of the minimal spanning tree for each filament of the sample.

The PDF, $N_{\text {fil }}$, is well fitted by

$N_{\text {fil }}=a_{0} \operatorname{erf}^{4}\left[a_{1}\left(x-x_{0}\right)\right] \exp \left(-\sqrt{a_{2}\left(x-x_{0}\right)}\right)$,

where $x=\Sigma_{\text {fil }} /\left\langle\Sigma_{\text {fil }}\right\rangle$ and

$a_{0}=50, \quad a_{1}=2.5, \quad a_{2}=27, \quad x_{0}=0.3$,

$a_{0}=180, \quad a_{1}=2, \quad a_{2}=42, \quad x_{0}=0.35$,

$a_{0}=60, \quad a_{1}=2.5, \quad a_{2}=31, \quad x_{0}=0.35$,

for these samples, respectively. Here the cut-off of the PDF at $x=x_{0}$ appears because we cannot select filaments with $\Sigma_{\text {fil }} \leq 1 / r_{\text {lnk }}$. This cut-off increases $\left\langle\Sigma_{\text {fil }}\right\rangle$ and changes the parameters $a_{0}, a_{1}, a_{2}$ in Eq. (64) with respect to the expected ones Eq. (63). This means that the measured mean linear density, $\left\langle\Sigma_{\text {fil }}\right\rangle$, depends upon the linking length used for the selection of filaments, and for the samples used we have

$r_{\text {lnk }} \Sigma_{\text {fil }}=3.4, \quad r_{\text {lnk }} \Sigma_{\text {fil }}=3.1, \quad r_{\text {lnk }} \Sigma_{\text {fil }}=3.1$, 
respectively. At the same time, for all samples at $\Sigma_{\text {fil }} \geq\left\langle\Sigma_{\text {fil }}\right\rangle$ the exponential term dominates, what is consistent with theoretical expectations (63).

For the surface density of filaments, $\left\langle\sigma_{\text {fil }}\right\rangle$, and for their mean separation, $\left\langle D_{\text {fil }}\right\rangle=\left\langle\sigma_{\text {fil }}\right\rangle^{-1 / 2}$ we get, respectively:

$\left\langle\sigma_{\mathrm{fil}}\right\rangle \approx \frac{W_{\mathrm{f}}\left(r_{\mathrm{lnk}}\right)\langle n\rangle}{(1+z)^{2}\left\langle\Sigma_{\mathrm{fil}}\right\rangle},\left\langle D_{\mathrm{fil}}\right\rangle=\sqrt{\frac{(1+z)^{2}\left\langle\Sigma_{\mathrm{fil}}\right\rangle}{W_{\mathrm{f}}\left(r_{\text {lnk }}\right)\langle n\rangle}}$.

Here $W_{\mathrm{f}}\left(r_{\text {lnk }}\right)$ and $\langle n\rangle$ are the fraction of objects accumulated by filaments selected with a given $r_{\text {lnk }}$ and the mean density of objects in the sample. Evidently, $W_{\mathrm{f}} \ll 1$ for $r_{\text {lnk }}\langle n\rangle^{1 / 3} \leq 1$ and $W_{\mathrm{f}} \rightarrow 0.3-0.5$ for larger $r_{\text {lnk }}$. The variations of $W_{\mathrm{f}}$ strongly influence $\left\langle\sigma_{\text {fil }}\right\rangle$ and $\left\langle D_{\text {fil }}\right\rangle$ and determine how they vary with $r_{\text {lnk }}$, which is strongly connected with the overdensity of selected filaments. However, these estimates of $\left\langle\sigma_{\text {fil }}\right\rangle$ do not take into account possible accumulation of filaments by walls and therefore they should be corrected by comparing them with observations and simulations.

\section{Characteristics of walls and pancakes}

From the PDF of the differences of displacements (19) it is possible to extract important approximate characteristics of walls and less massive pancakes which can be directly compared with available observations. Some of them were introduced in DD99 and successfully compared with the simulated and observed characteristics of walls. Here we derive the theoretical expression for the mean linear number density of pancakes for different redshifts and show how the transverse compression and expansion of pancakes after their formation change their observed characteristics. These results are successfully used for description of low mass pancakes observed as Ly- $\alpha$ forest at large redshifts (Demiański et al. 2003) and for the determination of the initial power spectrum at small scale (Demiański \& Doroshkevich 2003).

\subsection{Distribution function of wall sizes}

The distribution function of sizes of walls describes their frequency distribution with respect to their Lagrangian size what is identical to their surface density, $m_{\mathrm{w}}$, defined as the mass per unit surface area of the wall at the moment of its formation. This distribution function is obtained by integrating Eq. (30) over all $q_{2}$ and $q_{3}$ for $q_{0} \ll 1, q \leq 1$ and it is given by:

$N_{\mathrm{w}} \approx \frac{1}{\sqrt{2 \pi} \tau} \frac{\Theta_{\mathrm{w}}\left(q_{0} / q_{\mathrm{w}}\right)}{\sqrt{q_{\mathrm{w}}}} \exp \left(-\frac{q_{\mathrm{w}}}{8 \tau^{2}}\right) \operatorname{erf}\left(\sqrt{\frac{q_{\mathrm{w}}}{8 \tau^{2}}}\right)$,

$q_{\mathrm{w}}=\frac{m_{\mathrm{w}}}{l_{v}\langle\rho\rangle}=\frac{\left|\boldsymbol{q}_{1}-\boldsymbol{q}_{2}\right|}{l_{v}}, \quad \int_{0}^{\infty} N_{\mathrm{w}}\left(q_{\mathrm{w}}\right) \mathrm{d} q_{\mathrm{w}}=1$,

$\left\langle q_{\mathrm{w}}\right\rangle=\int_{0}^{\infty} q_{\mathrm{w}} N_{\mathrm{w}}\left(q_{\mathrm{w}}\right) \mathrm{d} q_{\mathrm{w}} \approx 8(0.5+1 / \pi) \tau^{2} \approx 6.55 \tau^{2}$,

$\Theta_{\mathrm{w}}(y)=4 \sqrt{q_{\mathrm{w}}} \mathrm{d} \mu\left(q_{\mathrm{w}}\right) / \mathrm{d} q_{\mathrm{w}}$.

The factors $\operatorname{erf}\left(\sqrt{q_{\mathrm{w}} / 8 \tau^{2}}\right)$ and $\Theta_{\mathrm{w}}$ in Eq. (66) describe the survival probability of walls and the influence of the coherent length of initial density field, $q_{0}$. For $q_{\mathrm{w}} \gg q_{0}, \Theta_{\mathrm{w}}=1$ and $N_{\mathrm{w}}$ becomes identical to the one found in DD99.

\subsection{Transverse characteristics of walls and pancakes}

For some applications we have to estimate the transverse characteristics of pancakes such as the distribution function of Lagrangian size and the mean real size of pancakes. These characteristics can be found with the method used above.

For the frequency distribution of the walls with Lagrangian transverse sizes, $q_{2}$ and $q_{3}$, we get from Eqs. (20), (21) and (30)

$N\left(\eta_{2}\right) \mathrm{d} \eta_{2}=\frac{2}{\sqrt{\pi}} \exp \left(-\eta_{2}^{2}\right) \mathrm{d} \eta_{2}, \quad \eta_{0} \leq \eta_{2} \leq \infty$,

$\left\langle\eta_{2}\right\rangle=1 / \sqrt{\pi}, \quad\left\langle\eta_{2}^{2}\right\rangle=\left\langle q_{2}\right\rangle / 8 \tau^{2}=1 / 2$,

and the same distribution for $\eta_{3}$ and $q_{3}$. In these cases, for the low mass objects the merging and percolation are not so important and the functions (67) are not corrected for the survival probability.

The mean transverse size of expanded, $\Delta r_{\mathrm{e}}$, and compressed, $\Delta r_{\mathrm{c}}$, pancakes can be found from relations (10), (19) and (67), we have

$\left\langle\Delta r_{\mathrm{e}}\right\rangle=\frac{\Delta_{0}}{2}\left(1+\frac{1}{\pi}\right), \quad\left\langle\Delta r_{\mathrm{e}}^{2}\right\rangle=\frac{7 \Delta_{0}^{2}}{8}\left(1+\frac{8}{7 \pi}\right)$,

$\left\langle\Delta r_{\mathrm{c}}\right\rangle \approx \frac{\Delta_{0}}{2}\left(1+\frac{1}{2 \pi}\right), \quad\left\langle\Delta r_{\mathrm{c}}^{2}\right\rangle \approx \frac{13 \Delta_{0}^{2}}{16}\left(1+\frac{10}{13 \pi}\right)$,

$\Delta_{0}=\frac{8 \tau^{2}}{1+z} l_{v}$

At small redshifts for the $\Lambda \mathrm{CDM}$ cosmological model with $\Gamma=0.2,8 \tau_{0}^{2} \approx 0.5$, the expected mean transverse size of walls $\sim 0.5 l_{v} \approx 16 h^{-1} \mathrm{Mpc}$ is similar to the observed one (Doroshkevich et al. 2001).

\subsection{Distribution function of pancakes surface density}

After pancake formation, the transverse compression and/or expansion of matter changes its surface density and other characteristics. However, the direct analysis indicates that the PDF of pancakes surface density given by Eq. (66) only weakly depends upon these deformations.

The evolution of the pancake surface area, $S_{\text {pan }}$, is described by the relation (10) as follows:

$S_{\mathrm{pan}}(z) \propto \frac{\Delta r_{2} \Delta r_{3}}{q_{2} q_{3}}=\frac{1}{(1+z)^{2}}\left(1-\frac{s_{2}}{\sqrt{2} \eta_{2}}\right)\left(1-\frac{s_{3}}{\sqrt{2} \eta_{3}}\right)$.

Therefore, for a pancake formed at a redshift $z_{\mathrm{f}}$ with the surface density

$\sigma_{\mathrm{pan}}\left(z_{\mathrm{f}}\right)=l_{v}\left(1+z_{\mathrm{f}}\right)^{2} q_{1}$,

the surface density at a redshift $z$ is

$\sigma_{\mathrm{pan}}(z)=\sigma_{\mathrm{pan}}\left(z_{\mathrm{f}}\right) S_{\mathrm{pan}}\left(z_{\mathrm{f}}\right) / S_{\mathrm{pan}}(z)$.

This means that the fraction of matter accumulated by pancakes with the surface density $\geq \sigma_{\text {pan }}$ depends upon their transversal 
sizes, $q_{2}$ and $q_{3}$, and instead of Eq. (26) we get from Eq. (19):

$$
\begin{aligned}
W_{\sigma}= & \frac{3}{\pi} \int_{-\infty}^{\eta_{2}, \eta_{3}} \mathrm{~d} x_{2} \mathrm{~d} x_{3} \exp \left(-x_{2}^{2}-x_{3}^{2}\right) \\
& \times \operatorname{erfc}\left(-\eta_{\mathrm{p}} \sqrt{\frac{\left[1-x_{2} / \eta_{2}(z)\right]\left[1-x_{3} / \eta_{3}(z)\right]}{\left[1-x_{2} / \eta_{2}\left(z_{\mathrm{f}}\right)\right]\left[1-x_{3} / \eta_{3}\left(z_{\mathrm{f}}\right)\right]}}\right) .
\end{aligned}
$$

As before, here $\mathrm{d}^{3} W_{\sigma} / \mathrm{d}^{3} q_{i}$ is the PDF similar to Eq. (30) and, after integration of this PDF over all $q_{2}, q_{3}$, we obtain Eq. (70) with $q_{2}, q_{3} \rightarrow \infty$. So, we get

$$
N_{\sigma} \rightarrow \exp \left(-\eta_{\mathrm{p}}^{2}\right) \frac{\mathrm{d} \eta_{\mathrm{p}}}{\mathrm{d} \sigma_{\mathrm{pan}}}, \quad \eta_{\mathrm{p}}^{2}=\frac{\sigma_{\mathrm{pan}}}{8 \tau^{2}(1+z)^{2}} .
$$

If the survival probability of a pancake is $\operatorname{erf}\left(\eta_{\mathrm{p}}\right)$ then the normalized PDF of surface density is given by Eq. (66), where $q_{\mathrm{w}}$ and $\tau$ are replaced by $\sigma_{\text {pan }}$ and $\tau_{z}=(1+z) \tau$, and, for example,

$$
\left\langle\sigma_{\text {pan }}\right\rangle=8(0.5+1 / \pi)(1+z)^{2} \tau^{2} .
$$

However, if we retain the expression for the survival probability used in Eq. (66) then the statistical characteristics of pancakes will depend upon both the redshift of pancake's formation and its current redshift.

These results indicate that at high redshifts, when $(1+$ $z) \tau(z) \approx$ const. the expansion and compression of pancakes approximately compensate each other and the PDF Eqs. (62) or (66), the mean surface density, $\left\langle\sigma_{\text {pan }}\right\rangle$, and other average characteristics of pancakes only weakly depend upon the redshift. They indicate also that, in spite of the strong evolution of each individual pancake, the statistical description (66) remains valid also when we consider each wall and each pancake as formed at their current redshifts.

Application of these results to absorbers observed in a wide range of redshifts (Demiański et al. 2003) confirms these conclusions.

\subsection{Mean comoving linear number density of walls}

Using the relations (30) and (66) it is also possible to obtain an approximate estimate of the mean comoving linear number density of recently formed walls, that is the mean number of walls per unit distance along a straight line. For richer walls with a threshold surface density $q_{\mathrm{thr}} \gg q_{0}$ the small scale fluctuations of density are not important and this function can be written as follows:

$$
\begin{aligned}
& \left\langle n_{\mathrm{w}}\left(\geq q_{\mathrm{thr}}\right)\right\rangle \approx \frac{3}{8} \frac{\operatorname{erfc}\left(\eta_{\mathrm{thr}}\right)}{l_{v}} \frac{(1+z)^{2}}{\left\langle q_{\mathrm{w}}\left(q_{\mathrm{thr}}\right)\right\rangle}, \\
& \left\langle q_{\mathrm{w}}\right\rangle=4 \tau^{2}\left[1+\frac{4 \sqrt{\pi} \eta_{\mathrm{thr}} \operatorname{erf}\left(\eta_{\mathrm{thr}}\right)+2 \exp \left(-\eta_{\mathrm{thr}}^{2}\right)}{\pi \exp \left(\eta_{\mathrm{thr}}^{2}\right) \operatorname{erfc}\left(\eta_{\mathrm{thr}}\right)\left[1+\operatorname{erf}\left(\eta_{\mathrm{thr}}\right)\right]}\right],
\end{aligned}
$$

where $\eta_{\mathrm{thr}}^{2}=q_{\mathrm{thr}} / 8 \tau^{2}$, and the factor $(1+z)^{2}$ describes the expansion of the universe. For $q_{\mathrm{thr}} \rightarrow 0, \eta_{\mathrm{thr}} \ll 1$, we have $\left\langle q_{\mathrm{w}}\left(q_{\mathrm{thr}}\right)\right\rangle \rightarrow\left\langle q_{\mathrm{w}}(0)\right\rangle$ as given by Eq. (66) and the mean linear number density of pancakes increases as

$\left\langle n_{\mathrm{w}}\left(\geq q_{\mathrm{thr}}\right)\right\rangle \propto(1+z)^{2} \tau^{-2} \propto(1+z)^{4}$.
For $\eta_{\mathrm{thr}} \gg 1$ we have $\left\langle q_{\mathrm{w}}\left(q_{\mathrm{thr}}\right)\right\rangle \approx q_{\mathrm{thr}}$. Similar relations can also be written for the threshold surface density of pancakes. Application of these results to the Lyman- $\alpha$ clouds observed in a wide range of redshifts (Demiański et al. 2003; Demiański $\&$ Doroshkevich 2003) confirms that this relation correctly describes the observed redshift distribution of stronger lines in the Ly- $\alpha$ forest.

\subsection{Coagulation approach}

To describe the nonlinear evolution of walls observed in deep galaxy surveys we can also use the $1 \mathrm{D}$ version of the coagulation equation (Smoluchowski 1916; Silk \& White 1978) which can be written in the comoving space as follows:

$$
\begin{aligned}
\frac{\partial n(q, \tau)}{\partial \tau}= & \frac{1}{2} \int_{0}^{q} \mathrm{~d} x P(x, q-x, \tau) n(x, \tau) n(q-x, \tau) \\
& -n(q, \tau) \int_{0}^{\infty} \mathrm{d} x P(x, q, \tau) n(x, \tau) .
\end{aligned}
$$

Here $n(q, \tau)$ is the comoving linear number density of walls with the dimensionless surface density $q, P(x, q, \tau)$ is the rate of aggregation of walls, and it is assumed that walls accumulate the main fraction of mass. Using this approach it is possible to find the linear number density of walls but their evolution depends on the unknown aggregation rate $P(x, q, \tau)$ which is a complicated function of $q$ and $\tau$. In particular, it depends upon the initial power spectrum.

The simplest reasonable solution of the coagulation equation, similar to Eq. (66), can be written as follows:

$n(q, \tau)=\frac{16}{l_{v} P_{0}^{2} \tau^{4}} \exp \left(-\frac{4 q}{P_{0} \tau^{2}}\right), \quad P(x, q, \tau)=P_{0} \tau$

and for the mean linear number density of walls we get:

$$
\begin{aligned}
\left\langle n\left(q_{\mathrm{thr}}, \tau\right)\right\rangle & =(1+z)^{2} \int_{q_{\mathrm{thr}}}^{\infty} \mathrm{d} x n(x, \tau) \\
& =\frac{4(1+z)^{2}}{l_{v} P_{0} \tau^{2}} \exp \left(-\frac{4 q_{\mathrm{thr}}}{P_{0} \tau^{2}}\right),
\end{aligned}
$$

what is similar to Eq. (72). For more complicated aggregation rates $P(x, q, \tau)$ some solutions were given by Silk \& White (1978).

\subsection{Linear number density of low mass pancakes}

The approach discussed in Sect. 7.4 neglects the influence of small scale perturbations and approximately characterizes only the mean linear number density of richer walls. For low mass pancakes of a size comparable with the coherent scale of initial density field, $q_{\mathrm{w}} \sim q_{0}$, the mean linear number density of pancakes with a threshold size $q_{\text {thr }}$ depends upon the spectral moment, $m_{0}$, and $q_{0}(4)$. It can be found with the standard 
technique (see, e.g., BBKS) used to describe the condition that a random function exceeds a certain value, we get:

$\left\langle n\left(\geq q_{\mathrm{thr}}\right)\right\rangle \approx \frac{\sqrt{3}(1+z)^{2}}{16 \pi l_{v} \tau(z) \sqrt{q_{0}}} \Theta\left(q_{\mathrm{thr}}\right) \Phi\left(\eta_{\mathrm{thr}}, \eta_{2}, \eta_{3}\right)$,

$\Phi=\frac{\operatorname{erf}\left(\eta_{\mathrm{thr}}\right)}{\eta_{\mathrm{thr}}} \int_{-\infty}^{\eta_{2}, \eta_{3}} \frac{\mathrm{d} x_{2} \mathrm{~d} x_{3}}{\pi} \exp \left(-\eta_{\mathrm{thr}}^{2}-x_{2}^{2}-x_{3}^{2}\right)$,

$\Theta^{2}\left(q_{\mathrm{thr}}\right)=1+\frac{q_{0}}{3} \frac{\mathrm{d}^{2} \xi_{v}}{\mathrm{~d}^{2} q}, \quad \eta_{\mathrm{thr}}^{2}=q_{\mathrm{thr}} / 8 \tau^{2}(z)$

Here the factors $(1+z)^{2}$ and $\operatorname{erf}\left(\eta_{\text {thr }}\right)$ describe the impact of expansion of the universe and merging of pancakes. The factor $\Theta$ in Eq. (76) introduces corrections for the case $q \leq q_{0}$, while $\Theta \rightarrow 1$, for $q \gg q_{0}, y \gg 1$. The density (76) depends upon transverse motions characterized by the parameters $\eta_{2}$ and $\eta_{3}$. For $\eta_{2}$ and $\eta_{3} \gg 1, \eta_{\text {thr }} \ll 1,\left\langle n\left(\geq q_{\text {thr }}\right)\right\rangle \propto(1+z)^{3}$ and it grows not so fast as for the richer walls (72) because formation of pancakes with $q \leq q_{0}$ is suppressed.

The relation (76) characterizes pancakes by their threshold size at the redshift of formation and neglects evolution of pancakes after they are formed. However, the surface density of formed pancakes is changing because of their transversal compression and/or expansion which shifts some of the pancakes under and/or over the observational threshold. This problem is quite similar to that discussed in Sect. 7.3, where it was noticed that the surface density is a more adequate characteristic of DM pancakes because it takes into account these variations.

In the relations (76) the threshold size of pancakes, $q_{\mathrm{thr}}$, together with their transverse sizes appears only in the function $\Phi\left(\eta_{\mathrm{thr}}, \eta_{2}, \eta_{3}\right)$. This means that to go from the threshold size to the threshold surface density we must link $q_{\mathrm{thr}}$ and $\sigma_{\mathrm{thr}}$ with the expression (69) and find a new function $\Phi\left(\sigma_{\text {thr }}, \eta_{2}, \eta_{3}\right)$. This procedure is quite similar to that used in Sect. 7.3, and, for $\eta_{2} \rightarrow \infty, \eta_{3} \rightarrow \infty$, we get instead of Eq. (76) that

$$
\begin{aligned}
\left\langle n\left(\geq \sigma_{\mathrm{thr}}\right)\right\rangle & \approx \frac{\sqrt{3}(1+z)^{2} \Theta}{16 \pi l_{v} \tau(z) \sqrt{q_{0}}} \exp \left(-\eta_{s}^{2}\right) \frac{\operatorname{erf}\left(\eta_{s}\right)}{\eta_{s}}, \\
\eta_{s}^{2} & =\frac{\sigma_{\mathrm{thr}}}{8 \tau^{2}(1+z)^{2}} .
\end{aligned}
$$

This result demonstrates again that, as was discussed in Sect. 7.3, the transverse compression and/or expansion of pancakes compensate each other and it does not change their statistical characteristics, if we consider each pancake as formed at its current redshift.

Both expressions (76) and (77) were used in Demiański et al. (2003) to describe the observed evolution of the mean linear number density of pancakes and to estimate the important parameter $q_{0}$ and the moment $m_{0}$ of the initial power spectrum.

\section{Summary and discussion}

In this paper we continue the statistical description of the process of LSS formation and evolution based on the Zel'dovich theory of nonlinear gravitational instability. First results obtained in DD99, DDMT and Demiański et al. (2003) show a significant potential of this approach. Here we are allowing for deformation of pancakes after their collapse along the axis of the most rapid compression, interaction of large and small scale perturbations and the impact of small scale cutoff in the initial power spectrum. This extension allows one to consider three important problems.

First of all, we are able to approximate the mass functions and fractions of matter accumulated by the LSS elements, namely, pancakes, filaments and halos for a wide range of redshifts. As was shown in Sect. 6, the mass functions describe reasonably well simulated mass distributions at all redshifts what emphasizes the generic character of the processes of formation of all structure elements. These functions provide quantitative description of the LSS evolution what in itself is an important problem.

Secondly, we discuss the interaction of large and small scale perturbations, that manifests itself as a strong concentration of galaxies within filaments and walls observed at small redshifts. In Sect. 4 we demonstrate the importance of this interaction and can roughly quantify it. However, this interaction is complex and it requires more detailed statistical description.

Thirdly, we derive the mass function and the mean linear number density of pancakes at high redshifts. Both functions play an important role in the interpretation of the Ly- $\alpha$ forest observed in spectra of the farthest quasars. Results obtained in Sect. 7 are successfully applied in Demiański et al. (2003) and Demiański \& Doroshkevich (2003) for detailed description of the observed absorbers and, in particular, lead to estimates of the spectral moment $m_{0}$ and the mass of the dominant fraction of dark matter particles.

The rapid growth of the observed concentration of neutral hydrogen at redshifts $z \sim 6$ (Djorgovski et al. 2001; Becker et al. 2001; Pentericci et al. 2001; Fan et al. 2001) is an evidence in favor of the reionization of the Universe at this redshift. These observations stimulate discussions of the reheating of the universe and, in particular, of the warm dark matter (WDM) models (see, e.g., Barkana et al. 2001; Loeb \& Barkana 2001). This means that it is worthwhile to find direct estimates of the small scale initial power spectrum and its influence on the LSS formation. Results obtained in Sects. 4, 5 and 7 are quite important for such investigations.

\subsection{Amplitude of perturbations}

In the Zel'dovich theory the evolutionary stage achieved in the sample under investigation is suitably characterized by an effective dimensionless "time", $\tau\left(z, \Omega_{\mathrm{m}}, h\right)$, introduced by Eq. (13):

$\tau(z)=\tau_{0}\left(\Omega_{\mathrm{m}}, h\right) B\left(\Omega_{\mathrm{m}}, z\right), \quad \tau_{0}=\frac{\sigma_{s}}{\sqrt{3} l_{v}}=\frac{A m_{-2}^{3 / 2}}{\sqrt{3}}$.

The function $B(z)$, the amplitude $A$ and the spectral moment $m_{-2}$ were introduced in (2)-(4). Here we present results of measurements of the amplitude $\tau_{0}$ with different methods discussed in DDMT. More popular characteristics of the amplitude are $\sigma_{8}$ - the variance of mass in a randomly placed sphere of radius $8 h^{-1} \mathrm{Mpc}$, and the correlation function of observed or simulated matter distribution. 
The amplitude of initial perturbations, $A$, is simply linked with $\sigma_{8}$ and the main parameters of the cosmological model, $h$ and $\Omega_{\mathrm{m}}$. Using the latest estimates (Spergel et al. 2003; Tegmark et al. 2004) for the $\Lambda$ CDM model (2) we get:

$\sigma_{8} \approx 0.055 A \approx 0.9 \pm 0.1, \quad A \approx 16.4 \pm 1.82$

$\tau_{0}=\tau_{8} \approx(0.22 \pm 0.02)$

where $\tau_{8}$ is the amplitude $\tau_{0}$ deduced form $\sigma_{8}$.

The variance of displacement (7), $\sigma_{s}$ and $\tau$, can be directly expressed through the observed two point correlation function of galaxies, $\xi_{\text {gal }}(r)$. The correlation functions for the APM survey were found in Loveday et al. (1996), for the Las Campanas Redshift Survey in Jing et al. (1998) and for the Durham/UKST Redshift Survey in Ratcliffe et al. (1998). Using these results we get

$\tau_{0}=\tau_{\xi} \approx(0.2 \pm 0.04)\left(\frac{\Omega_{\mathrm{m}} h^{2}}{0.2}\right)$,

where $\tau_{\xi}$ is the amplitude $\tau_{0}$ deduced form the correlation function of galaxies.

Applying the relations (66) to the systems of walls selected in two largest redshift surveys, namely, the $2 \mathrm{dF}$ and the SDSS DR1 (Doroshkevich et al. 2004) allows one to estimate $\tau_{0}$ as

$\tau_{0}=\tau_{\mathrm{w}}=(0.24 \pm 0.02) \sqrt{\Omega_{\mathrm{m}} h^{2} / 0.2}$,

where $\tau_{\mathrm{w}}$ is the amplitude $\tau_{0}$ deduced from the observed properties of walls.

All these estimates are consistent with each other and the differences between $\tau_{8}, \tau_{\xi}$ and $\tau_{\mathrm{w}}$ given by (79)-(81) reflect the precision actually achieved in modern observations.

\subsection{Mass function of structure elements}

The Press-Schechter formalism derived for the spherical collapse describes quite well the mass functions of various observed and simulated structure elements. In spite of this, all attempts to extend it to the collapse of asymmetric objects failed with the exception of recently proposed description of collapse of elliptical clouds (see, e.g., Loeb \& Barkana 2001; Sheth \& Tormen 2002). In this paper we demonstrate that the Zel'dovich theory successfully describes collapse of any object.

Indeed, formation and relaxation of pancakes along the axis of the most rapid compression does not prevent their deformation in transverse directions due to relatively small gradients of density and pressure in these directions. The growth of density within the pancake changes the rate of evolution and accelerates its compression and further transformation into high density filaments and clouds. However, the masses of filaments and clouds formed due to such compression remain almost unchanged. The same factor also decelerates the expansion of the pancake and its dissipation and, so, increases the fraction of surviving pancakes.

This means that, allowing for these deformations, we obtain approximate time dependent mass functions of structure elements formed due to successive compression in one, two and three directions. These results emphasize the generic character of the formation of all structure elements and link the fundamental characteristics of structure with the initial power spectrum. They extend the Press-Schechter formalism for all LSS elements including the filaments and walls which are far from equilibrium. The unexpected similarity of the mass functions for these elements verifies that the shape of collapsed clouds influences the rate of collapse but it does not change significantly their mass functions.

As is seen from the comparison of results presented in Sect. 5 and in Loeb \& Barkana (2001) for the Press-Schechter formalism, the mass functions derived in both approaches are similar and, in particular, the relations (48) and (54) resemble each other. Both mass functions are sensitive to the damping of small scale perturbations caused by the random motion of DM particles and the Jeans damping. They predict the existence of numerous low mass objects which can be identified with isolated dwarf galaxies and a rich population of Ly- $\alpha$ absorbers. Both approaches predict a strong suppression of formation of isolated low mass objects with $M \leq M_{\rho}$.

This comparison indicates that the differences between these approaches are quantitative rather than qualitative. The similarity of the mass functions demonstrates, in fact, the self similar character of the process of structure evolution that is the successive condensation of matter within clouds, filaments and walls with progressively increased sizes and masses. At the same time, the approximate character of both the Zel'dovich theory and the Press-Schechter formalism implies that the proposed mass functions are only approximate. Detailed analysis of high resolution simulations with application of the approach proposed in this paper allows one to improve results obtained in Sect. 5.

Due to their high overdensity above the mean density, both filaments and walls are easily detected in the deep galaxy surveys using the Minkowski Functional approach (Schmalzing et al. 1999; Kerscher 2000) and the well known friend-of-friend method generalized in the Minimal Spanning Tree technique (Barrow et al. 1985; van de Weygaert 1991; Doroshkevich et al. 2001, 2002). The mass functions of the observed structure elements can be compared with the expected ones. For simulations the same comparison can be performed at all redshifts what allows one to trace the expected redshift dependence of these functions.

In Sect. 5.7 for the first time we compare the expected mass functions with simulated ones. In Doroshkevich et al. (2004) the expected mass functions are compared with the observed ones for the LSS elements selected with various threshold overdensity from the SDSS EDR. These results show that both relations (47) and (54) describe quite well even the mass distribution of filaments and walls with a moderate richness. Stronger disagreement appears for the richest walls and filaments formed due to the process of percolation that is not described by the Zel'dovich theory.

However, the potential of both approaches is limited as they cannot describe incorporation of filaments and walls into a joint network (percolation process) and the final disruption of collapsed clouds what leads to formation of numerous low mass satellites of the central object. Neither the Press-Schechter 
formalism nor the Zel'dovich theory can describe the faster evolution of clouds accumulated by richer walls as compared with isolated clouds. As was discussed in Sect. 5, both approaches do not describe the coagulation of high density clouds what distorts their mass function and makes it similar to the power low. These problems remain open for further investigations.

\subsection{Interaction of large and small scale perturbations. Large scale bias}

The analysis of redshift distribution of absorbers shows almost homogeneous spatial distribution of baryonic and DM components of the matter on scales $\geq 1 h^{-1} \mathrm{Mpc}$. At the same time, the observed spatial distribution of luminous matter is strongly inhomogeneous. Thus, about half of the observed galaxies are concentrated within large walls while, for example, within the Böotes void the number of galaxies is very small. The observed walls and voids are associated with compressed and expanded regions of the Universe and these observations point out to the possible correlations between the galaxy formation and the large scale deformation field (see, e.g., Rees 1985; Dekel \& Silk 1986; Dekel \& Rees 1987). Some of such correlations were already noticed in simulations (see, e.g., Sahni et al. 1994).

As was shown in Sect. 4, the Zel'dovich theory allows one to quantify this interaction and demonstrates that the formation rate of high density objects is modulated by large scale perturbations. This modulation cannot significantly change the redshift evolution of the fraction of mass accumulated by structure elements. However, this interaction accelerates the formation of high density halos and galaxies within regions associated with the future LSS elements such as clusters of galaxies, filaments and walls. These results suggest that the poorer sample of isolated galaxies and invisible DM halos situated within low density regions were formed later then similar galaxies and DM halos situated within filaments and walls. The results presented in Sect. 4.3 illustrate this influence for simulated matter distribution. This problem was widely discussed during last years (see, e.g., Hoffman et al. 1992; Little \& Weinberg 1994; Mo \& White 1996; Kauffmann et al. 1999a,b,c; Benson et al. 2001; Mathis \& White 2002). The same effect was also found in Gottlöber et al. (2001, 2002, 2003) for simulated spatial distribution of halos and in Doroshkevich et al. (2004) for properties of observed groups of galaxies.

\subsection{Properties of low mass pancakes and the mass of DM particles}

Numerous simulations performed recently (see, e.g., Zhang et al. 1998; Weinberg et al. 1999) demonstrate that the absorption lines observed in spectra of the farthest quasars - the Ly- $\alpha$ forest - are related to the numerous low mass clouds formed at high redshifts. As was discussed in Demiański et al. (2003), some of the statistical characteristics of observed absorbers can be successfully explained on the basis of Zel'dovich theory. At the same time, the analysis shows that the approximate description based on results obtained in DD99 have to be essentially improved. Indeed, the absorbers are observed in a wide range of redshifts and the properties of long-lived absorbers are changing with time at least due to their transverse expansion and compression.

Discussion of this problem in Sect. 7 shows that for statistically homogeneous sample of absorbers we can neglect this influence, at least for their two important characteristics, namely, for the PDF of their surface density (66) and their mean linear number density (76). Results obtained in Sect. 7 link these characteristics with the properties of the initial power spectrum at small scale. This means that the analysis of these characteristics allows one to measure the variance of initial density perturbations and to restrict the mass of dominant fraction of dark matter particles.

Results obtained in Sect. 7 were applied to study the Lyman- $\alpha$ forest in Demiański et al. (2003). This analysis confirms that the relations (66) and (76) describe quite well the PDF and the redshift distribution of $\sim 5000$ observed absorbers. These results also verify the Gaussianity of initial perturbations. Comparison of other characteristics of pancakes derived in Sects. 3 and 7 with observations can be found in Demiański et al. (2003). The shape of small scale initial power spectrum derived from the absorber characteristics was discussed in Demiański \& Doroshkevich (2003).

\subsection{Reheating of the universe}

The relations (28) and (46) show that in the Zel'dovich theory of gravitational instability the rate of matter collapse at high redshifts depends upon both the amplitude of perturbations, $\tau(z)$, as given by Eq. (13), and the coherent length of density field, $l_{\rho}$ and $q_{0}$, as given by Eq. (4), which, in turn, depends on the shape of the initial power spectrum at large $k$ and the mass of the dominant type of DM particles.

As was noticed in Sect. 3, the most effective matter condensation within high density clouds occurs at $\tau\left(z_{r}\right)=\tau_{r} \approx \sqrt{q_{0} / 6}$ (see Eq. (22)) when these clouds already accumulate $\sim 2 \%$ of matter and the main fraction of mass is concentrated within clouds with $M \sim(0.2-0.5)\langle M\rangle$.

For $q_{0} \sim 10^{-2}$ we can estimate the redshift of the period of most efficient condensation and the mean mass of DM clouds as follows:

$\tau_{r} \sim 0.04, \quad 1+z_{r} \approx 1.3 \tau_{0} / \tau_{r} \sim 7-8$,

$\left\langle M_{\mathrm{DM}}\right\rangle \sim \tau_{r}^{3} M_{v} \approx 10^{12} M_{\odot}\left(\frac{0.3}{\Omega_{\mathrm{m}}}\right)^{2}\left(\frac{0.7}{h}\right)^{4}$,

what is similar to the mass of a typical galaxy. For $q_{0} \sim 10^{-3}$ we have, respectively,

$\tau_{r} \sim 0.013, \quad 1+z_{r} \approx 1.3 \tau_{0} / \tau_{r} \sim 20$

$\left\langle M_{\mathrm{DM}}\right\rangle \sim 4 \times 10^{-2} \tau_{r}^{3} M_{v} \approx 10^{9} M_{\odot}\left(\frac{0.3}{\Omega_{\mathrm{m}}}\right)^{2}\left(\frac{0.7}{h}\right)^{4}$,

what is similar to the mass of a typical dwarf galaxy. These results illustrate strong links between the period of reheating and 
the shape of the power spectrum at small scale characterized by $q_{0}$. Even at higher redshifts noticeable fraction of matter can be compressed within high density clouds with masses of galactic scales.

According to available estimates (Haiman \& Loeb 1999; Loeb \& Barkana 2001) the reionization of the universe occurs after $\sim 1-3 \%$ of matter is concentrated within high density halos. From Eqs. (82) and (83) it follows that this fraction of collapsed matter can be reached already at $\tau \sim \tau_{r}$ at redshifts $z=z_{r} \sim 8-25$, for $q_{0} \sim 10^{-2}-10^{-3}$. The effective reionization and reheating of the universe at such redshifts is consistent with the observed concentration of neutral hydrogen at redshifts $z \sim 6$, which characterizes mainly the rate of generation and achieved intensity of UV background. This means that these ranges of $q_{0}$ and $z_{r}$ provide a reasonable estimate of the period of reheating.

The observations of environment of high redshift quasars (Fan et al. 2001) provide an evidence in favor of reheating of the Universe at $z \sim 6-10$, what is more consistent with $q_{0} \sim 10^{-2}$. However, our analysis shows that, for the standard WDM model with the Harrison - Zel'dovich large scale power spectrum and exponential cutoff caused by a mass $M_{\mathrm{DM}} \sim$ $1 \mathrm{keV}$ of the dominant fraction of DM particles, some problems can appear with formation of low mass isolated galaxies with $M \leq 10^{8}-10^{9} M_{\odot}$. Perhaps, some excess of power at small scales can help to solve this problem. First observational indications of such excess are presented in Demiański \& Doroshkevich (2003).

Acknowledgements. A.D. thanks V. Lukash and E. Mikheeva for useful discussions. This paper was supported in part by Denmark's Grundforskningsfond through its support for an establishment of Theoretical Astrophysics Center and a grant 1-P03D-014-26 of the Polish State Committee for Scientific Research. This work was supported in part by EC network HPRN-CT-2000-00124. A.D. also wishes to acknowledge support from the Center for Cosmo-Particle Physics "Cosmion" in the framework of the project "Cosmoparticle Physics". Furthermore, we wish to thank the anonymous referee for valuable discussion and many useful comments.

\section{References}

Abazajian, K. 2003, AJ, 126, 2081

Babul, A., \& Starkman, G. D. 1992, ApJ, 401, 28

Bardeen, J. M., Bond, J. R., Kaiser, N., \& Szalay, A. 1986, ApJ, 304, 15 (BBKS)

Barkana, R., Haiman, Z., \& Ostriker, J. P. 2001, ApJ, 558, 482

Barrow, J. D., Bhavsar, S. P., \& Sonoda, D. 1985, MNRAS, 216, 17

Becker, R. H., Fan, X., White, R. L., et al. 2001, AJ, 122, 2850

Benson, A. J., Frenk, C. S., Baugh, C. M., Cole, S., \& Lacey, C. G. 2001, MNRAS, 327, 1041

Bond, J. R., Cole, S., Efstathiou, G., \& Kaiser, N. 1991, ApJ, 379, 440

Bond, J. R., \& Myers, S. T. 1996a, ApJS, 41, 63

Bond, J. R., \& Myers, S. T. 1996b, ApJS, 103, 1

Bond, J. R., Kofman, L., \& Pogosian, D. 1996, Nature, 380, 603

Bullock, J. S., Dekel, A., Kollat, T. S., et al. 2001, ApJ, 555, 240

Bunn, E. F., \& White, M. 1997, ApJ, 480, 6

Cen, R. 1994, ApJ, 424, 22

Cole, S., Hatton, S., Weinberg, D. H., \& Frenk, C. S. 1998, MNRAS, 300,945
Colless, Peterson, B. A., Jackson, C., et al. 2003

[arXiv: astro-ph/0306581]

Dave, R., Hernquist, L., Katz, N., \& Weinberg, D. ApJ, 511, 521

Dekel, A., \& Silk, J. 1986, ApJ, 303, 39

Dekel, A., \& Rees, M. J. 1987, Nature, 326, 455

de Lapparent, V., Geller, M. J., \& Huchra, J. P. 1986, ApJ, 302L, 1

Demiański, M., \& Doroshkevich, A. 1999, MNRAS, 306, 779, (DD99)

Demiański, M., Doroshkevich, A., Müller, V., \& Turchaninov, V. I. 2000, MNRAS, 318, 1177 (DDMT)

Demiański, M., Doroshkevich, A., \& Turchaninov, V. I. 2003, MNRAS, 340, 525

Demiański, M., \& Doroshkevich, A. 2003, ApJ, 597, 81

Djorgovski, S. G., Castro, S., Stern, D., \& Mahabal, A. A. 2001, ApJ, 560, L5

Doroshkevich, A. 1970, Astrofisika, 6, 581

Doroshkevich, A. G. 1980, SvA, 24, 152

Doroshkevich, A., Tucker, D. L., Fong, R., Turchaninov, V., \& Lin, H. 2001, MNRAS, 322, 369

Doroshkevich, A., Tucker, D., Allam, S., \& Way, M. 2004a, A\&A, 418,7

Doroshkevich, A., Fong, R., Tucker, D., \& Way, M. 2004b, MNRAS, submitted

Einasto, J., Klypin, A., Saar, E., \& Shandarin, S. 1984, MNRAS, 206, 529

Epstein, R. I. 1983, MNRAS, 205, 207

Fan, X., Narayanan, Y. K., Strauss, M. A., et al. 2002, AJ, 123, 1247

Frenk, C. S. 2002 [arXiv: astro-ph/0208219]

Gottlöber, S., Klypin, A., \& Kravtsov, A. 2001, ApJ, 546, 223

Gottlöber, S., Kersher, M., Kravtsov, A., et al. 2002, A\&A, 387, 778

Gottlöber, S., Lokas, E., Klypin, A., \& Hoffman, Y. 2003, MNRAS, 344,715

Haiman, Z., \& Loeb, A. 1999, ApJ, 519, 479

Hoffman, Y., Silk, J., \& Wyse, R. 1992, ApJ, 388, 13

Hoyle, F. 1949, Problems of Cosmical Aerodynamics, 195

Jenkins, A., Frenk, C. S., Pearce, F. R., et al. 1998, ApJ., 499, 20

Jing, Y. P., Mo, H. J., \& Boerner, G. 1998, ApJ, 494, 1

Kauffmann, G., Golberg, J. M., Diaferio, A., \& White, S. D. M. 1999a, MNRAS, 303, 188

Kauffmann, G., Golberg, J. M., Diaferio, A., \& White, S. D. M. 1999b, MNRAS, 307, 529

Kauffmann, G., Golberg, J. M., Diaferio, A., \& White, S. D. M. 1999c, MNRAS, 307, 537

Kerscher, M. 2000, Lect. Notes Phys., 554, 11

Klypin, A., Gottlöber, S., Kravtsov, A. V., \& Khokhlov, A. M. ApJ, 516,530

Little, B., \& Weinberg, D. 1994, MNRAS, 267, 605

Lemson, G., \& Kauffmann, G. 1999, MNRAS, 302, L111

Loveday, J., Maddox, S. J., Efstathiou, G., \& Peterson, B. A. 1995, ApJ, 442, 457

Loeb, A., \& Barkana, R. 2001, ARA\&A, 39,19

Luo, S., Vishniac, E., \& Martel, H. 1996, ApJ, 468, 62

Martinez, V. J., \& Saar, E. 2002, Statistics of the galaxy distribution (Chapman and Hall/CRC Press, Boca Raton)

Mathis, H., \& White, S. D. M. 2002, MNRAS, 337, 1193

Matarrese, S., \& Mohayaee, R. 2002, MNRAS, 329, 37

Mecke, K. R., Buchert, T., \& Wagner, H. 1994, A\&A, 288, 697

Mo, H., \& White, S. D. M. 1996, MNRAS, 282, 347

Narayanan, V. K., Spergel, D. N., Dave, R., et al. 2000, ApJ, 543, L103

Oort, J. H. 1983, ARA\&A, 21, 373

Peacock, J. A., \& Heavens, A. F. 1990, MNRAS, 243, 133

Peebles, P. J. E. 1969, ApJ, 155, 393 
Pentericci, L., Fan, X., Rix, H.-W., et al. 2002, AJ, 123, 2151

Press, W. H., \& Schechter, P. 1974, ApJ, 187, 425

Rees, M. J. 1985, MNRAS, 213, 75

Sahni, V., Sathyaprakash, B. S., \& Shandarin, S. F. 1994, ApJ, 431, 20

Sahni, V., \& Coles, P. 1995, Phys. Rep., 262, 1

Scannapieco, E., \& Barkana, R. 2002, ApJ, 571, 585

Schmalzing, J., Gottlöber, S., Klypin A. A., \& Kravtsov, A. V. 1999, MNRAS, 309, 1007

Shandarin, S. 1983, SvA, 9, L104

Shandarin, S., \& Zel'dovich, Ya. B. 1989, Rev. Mod. Phys., 61, 185

Shandarin, S. F., Melott, A. L., McDavitt, K., et al. 1995, Phys. Rev. Lett., 75,7

Shandarin, S. F., Sheth, J. V., \& Sahni, V. 2003 [arXiv: astro-ph/0312110]

Shectman, S. A., Landy, S. D., Demler, A., et al. 1996, ApJ, 470, 172 Sheth, R., \& Pitman, J. 1997, MNRAS, 289, 66

Sheth, R., \& Lemson, G. 1999, MNRAS, 305, 946

Sheth, R., \& Tormen, G. 1999, MNRAS, 308, 119

Sheth, R., \& Tormen, G. 2002, MNRAS, 329, 61

Sheth, R., \& Jain, B. 2003, MNRAS, 345, 529

Sheth, R. 2003, MNRAS, 345, 1200

Sheth, R., \& van de Weygaert, R. 2003 [arXiv:astro-ph/0311260]
Silk, J., \& White, S. D. M. 1978, ApJ, 223, L59

Smith, R. E., Peacock, J. A., Jenkins, A., et al. 2003, MNRAS, 341, 1311

Smoluchowski, M. 1916, Phys. Z., 17, 557

Spergel, D. N., Verde, L., Peiris, H. V., et al. 2003, ApJS, 148, 175

Stoughton, S., Lupton, R. H., Bernardi, M., et al. 2002, AJ, 123, 485, 576

Tegmark, M., Strauss, M., Blanton, M., et al. 2004, Phys. Rev. D, 69, 103501

Thompson, L. A., \& Gregory, S. A. 1978, ApJ, 220, 809

Vishniac, E. T. 1983, ApJ, 274, 152

Vivitska, M., Klypin, A. A., Kravtsov, A. V., et al. 2002, ApJ, 581, 799 van de Weygaert, R. 1991, Ph.D. Thesis, University of Leiden

Weinberg, D. H., Burles, S., Croft, R. A. C., et al. 1998, in Evolution of Large Scale Structure: From Recombination to Garching, ed. A. J. Banday, R. K. Sheth, \& L. N. Da Costa, 346

White, S. D. M. 1984, ApJ, 286, 38

Zel'dovich, Ya. B. 1970, A\&A, 5, 20

Zel'dovich, Ya. B., Einasto, J., \& Shandarin, S. F. 1982, Nature, 300, 407

Zhang, Yu., Meiksin, A., Anninos, P., \& Norman, M. L. 1998, ApJ, 495, 63 
M. Demiański and A. G. Doroshkevich: Statistical characteristics of large scale structure, Online Material p 1

\section{Online Material}


M. Demiański and A. G. Doroshkevich: Statistical characteristics of large scale structure, Online Material p 2

\section{Appendix A: Correlation functions of initial perturbations}

In this appendix we present a few correlation and structure functions which describe the relative spatial distribution of important parameters of the initial perturbations. These functions have been introduced in DD99, where more details can be found.

The structure function of gravitational potential perturbations characterizes correlation of the gravitational potential at two points $\tilde{\boldsymbol{q}}_{1}$ and $\tilde{\boldsymbol{q}}_{2}$. As the power spectrum is a function of only the absolute value of wave number $|k|$, this structure function depends on $\tilde{q}_{12}=\left|\tilde{\boldsymbol{q}}_{1}-\tilde{\boldsymbol{q}}_{2}\right|$ and for the perturbations of gravitational potential we have

$$
\begin{aligned}
& 3 \frac{\left\langle\Delta \phi_{12} \Delta \phi_{34}\right\rangle}{l_{v}^{2} \sigma_{s}^{2}}=G_{0}\left(\tilde{q}_{14}\right)-G_{0}\left(\tilde{q}_{13}\right) \\
& +G_{0}\left(\tilde{q}_{23}\right)-G_{0}\left(\tilde{q}_{24}\right), \quad \tilde{q}_{i j}=\left|\tilde{\boldsymbol{q}}_{i}-\tilde{\boldsymbol{q}}_{j}\right|, \\
& \Delta \phi_{12}=\phi\left(\tilde{\boldsymbol{q}}_{1}\right)-\phi\left(\tilde{\boldsymbol{q}}_{2}\right), \quad \Delta \phi_{34}=\phi\left(\tilde{\boldsymbol{q}}_{3}\right)-\phi\left(\tilde{\boldsymbol{q}}_{4}\right), \\
& G_{0}(\tilde{q})=\frac{3}{\pi^{2} l_{v}^{2} \sigma_{s}^{2}} \int_{0}^{\infty} \frac{p(k)}{k^{2}}\left(1-\frac{\sin k \tilde{q}}{k \tilde{q}}\right) \mathrm{d} k
\end{aligned}
$$

Differentiations of this structure function give other structure functions:

$$
\begin{aligned}
& \frac{3\left\langle\Delta \phi\left(\tilde{\boldsymbol{q}}_{12}\right) S_{i}\left(\tilde{\boldsymbol{q}}_{3}\right)\right\rangle}{l_{v} \sigma_{s}^{2}}=\left(\tilde{\boldsymbol{q}}_{1}-\tilde{\boldsymbol{q}}_{3}\right)_{i} G_{1}\left(\tilde{q}_{13}\right)-\left(\tilde{\boldsymbol{q}}_{2}-\tilde{\boldsymbol{q}}_{3}\right)_{i} G_{1}\left(\tilde{q}_{23}\right), \\
& \frac{3\left\langle S_{i}\left(\tilde{\boldsymbol{q}}_{1}\right) S_{j}\left(\tilde{\boldsymbol{q}}_{2}\right)\right\rangle}{\sigma_{s}^{2}}=\delta_{i j} G_{1}\left(\tilde{q}_{12}\right)+\left(\tilde{\boldsymbol{q}}_{12}\right)_{i}\left(\tilde{\boldsymbol{q}}_{12}\right)_{j} G_{2}\left(\tilde{q}_{12}\right) \\
& G_{1}(x)=G_{0}^{\prime} / x, G_{2}(x)=G_{1}^{\prime} / x, G_{3}(x)=G_{2}^{\prime} / x \\
& G_{12}(x)=G_{1}(x)+x^{2} G_{2}(x)=G_{0}^{\prime \prime}=\left(x G_{1}\right)^{\prime},
\end{aligned}
$$

For the Harrison-Zel'dovich primordial power spectrum and for the CDM transfer function (BBKS), these functions can be approximated by

$$
\begin{aligned}
& G_{1}(x) \approx\left(1+q_{0}\right)\left[1+\sqrt{q_{0}^{2}+x^{2}}+a_{0} x^{2}\right]^{-1}, \\
& G_{12} \approx G_{1}^{2}\left(1+q_{0}\right)^{-1}\left[1-a_{0} x^{2}+q_{0}^{2} / \sqrt{q_{0}^{2}+x^{2}}\right], \\
& G_{1}(0)=G_{12}(0)=1, \quad G_{2}(0)=-\left[q_{0}\left(1+q_{0}\right)\right]^{-1} . \\
& x=\tilde{q} / l_{v}, \quad q_{0}=l_{\rho} / l_{v}, \quad a_{0}=5\left(l_{v} / L_{0}\right)^{2} \approx 0.3,
\end{aligned}
$$

where the typical scale $L_{0}$ is defined as

$L_{0}^{2}=3 \pi^{2} \int_{0}^{\infty} p(k)\left(1-\frac{\sin k L_{0}}{k L_{0}}\right) k^{-2} \mathrm{~d} k / \int_{0}^{\infty} p(k) \mathrm{d} k$,

and the scales $l_{v}$ and $q_{0}$ were introduced by Eq. (4).

\section{Appendix B: Characteristics of the deformation field}

The general deformation of a spherical cloud with a diameter $q=\tilde{q} / l_{v}$ can be suitably characterized by the dimensionless random scalar function

$\Theta(\boldsymbol{q})=\Theta(q, \theta, \phi)=\frac{\sqrt{3}[\boldsymbol{S}(\tilde{\boldsymbol{q}} / 2)-\boldsymbol{S}(-\tilde{\boldsymbol{q}} / 2)] \cdot \boldsymbol{q}}{\sigma_{s} q}$,

instead of the deformation tensor $d_{i k}$. Expansion of this function into spherical harmonics characterizes deformations of the cloud with a required accuracy. This problem is similar to the usual description of CMB anisotropy in terms of spherical harmonics, and we have:

$\Theta(q, \theta, \phi)=\sum_{l, m} a_{l m}(q) Y_{l, m}(\theta, \phi)$,

$Y_{l, m}(\theta, \phi)=(-1)^{m} i^{l} \sqrt{\frac{2 l+1}{4 \pi} \frac{(l-m) !}{(l+m) !}} P_{l}^{m}(\cos \theta) \mathrm{e}^{i m \phi}$,

$Y_{l,-|m|}(\theta, \phi)=(-1)^{l-m} Y_{l, m}(\theta, \phi), l \geq m \geq 0$,

$\left.\left.\left\langle\Theta \boldsymbol{q}_{1}\right) \Theta \boldsymbol{q}_{2}\right)\right\rangle=\Psi\left(q, \mu_{12}\right)=\sigma^{2}(q) \sum_{l} b_{l}^{2}(q) P_{l}\left(\mu_{12}\right)$

$$
\begin{aligned}
= & G_{1}\left(x_{1}\right)+G_{1}\left(x_{2}\right)-G_{12}\left(x_{1}\right)-G_{12}\left(x_{2}\right) \\
& +\mu_{12}\left[G_{1}\left(x_{2}\right)-G_{1}\left(x_{1}\right)+G_{12}\left(x_{1}\right)-G_{12}\left(x_{2}\right)\right]
\end{aligned}
$$

$\mu_{12}=\frac{\boldsymbol{q}_{1} \boldsymbol{q}_{2}}{q_{1} q_{2}}, \quad x_{1}=q \sqrt{\frac{1+\mu_{12}}{2}}, x_{2}=q \sqrt{\frac{1-\mu_{12}}{2}}$,

$\sigma^{2}(q) b_{l}^{2}(q)=\sum_{m=-l}^{l} \frac{\left\langle a_{l m}^{2}\right\rangle}{4 \pi}=\frac{2 l+1}{4 \pi}\left\langle a_{l}^{2}\right\rangle, \quad \sum_{l} b_{l}^{2}=1$,

$\Psi\left(q, \mu_{12}=1\right)=\sigma^{2}(q), \quad \Psi\left(q, \mu_{12}=0\right)=\sigma^{2}(q) r_{s}(q)$,

$\sigma^{2}(q)=2\left[1-G_{12}(q)\right], \quad r_{s}(q)=-\frac{q^{2}}{\sigma^{2}} G_{2}\left(\frac{q}{\sqrt{2}}\right)$.

Here $\sigma^{2}(q)$ and $r_{s}(q)$ are the dispersion and the coefficient of correlation of dimensionless orthogonal displacements, and the functions $G_{1}, G_{2}$ and $G_{12}$ were introduced in Appendix A. The correlation coefficient $r_{s}$ is a weak function of $q$ and for $q \ll$ $1, q_{0} \ll 1$, we have

$1 / 3 \leq r_{s} \leq 2^{-3 / 2}$,

what is consistent with the usual correlations of the components of deformation tensor.

The function $\Psi\left(q, \mu_{12}\right)$ is symmetrical with respect to the replacement $\mu_{12} \rightarrow-\mu_{12}$ and, therefore, $b_{l}^{2}=0$ for odd $l$. Using the relations (A.3) we have for the relative amplitude of even spherical harmonics, for the most interesting cases $q_{0} \ll q \ll 1$ and $q \ll q_{0} \ll 1$, respectively:

$b_{0}^{2} \approx 0.533, b_{2}^{2} \approx 0.381, b_{4}^{2} \approx 0.037, b_{6}^{2} \approx 0.014, \ldots$

$b_{0}^{2} \approx 0.55, \quad b_{2}^{2} \approx 0.44 \gg b_{4}^{2}, b_{6}^{2} \ldots$

These results justify the assumption used in DD99 to neglect higher order harmonics of perturbations with $l \geq 4$. 